\title{
A YANG-BAXTER EQUATION FOR METAPLECTIC ICE
}

\author{
BEN BRUBAKER, VALENTIN BUCIUMAS, AND DANIEL BUMP
}

\begin{abstract}
We will give new applications of quantum groups to the study of spherical Whittaker functions on the metaplectic $n$-fold cover of $\mathrm{GL}(r, F)$, where $F$ is a nonarchimedean local field. Earlier Brubaker, Bump, Friedberg, Chinta and Gunnells had shown that these Whittaker functions can be identified with the partition functions of statistical mechanical systems. They postulated that a Yang-Baxter equation underlies the properties of these Whittaker functions. We confirm this, and identify the corresponding Yang-Baxter equation with that of the quantum affine Lie superalgebra $U_{\sqrt{v}}(\widehat{\mathfrak{g l} l}(1 \mid n))$, modified by Drinfeld twisting to introduce Gauss sums. (The deformation parameter $v$ is specialized to the inverse of the residue field cardinality.)

For principal series representations of metaplectic groups, the Whittaker models are not unique. The scattering matrix for the standard intertwining operators is vector valued. For a simple reflection, it was computed by Kazhdan and Patterson, who applied it to generalized theta series. We will show that the scattering matrix on the space of Whittaker functions for a simple reflection coincides with the twisted $R$-matrix of the quantum group $U_{\sqrt{v}}(\widehat{\mathfrak{g l}}(n))$. This is a piece of the twisted $R$-matrix for $U_{\sqrt{v}}(\widehat{\mathfrak{g l}}(1 \mid n))$, mentioned above.
\end{abstract}

\section{INTRODUCTION}

The formula of Casselman and Shalika [14 expresses values of the spherical Whittaker function for a principal series representation of a reductive algebraic group over a $p$-adic field in terms of the characters of irreducible finite-dimensional representations of the Langlands dual group. Their proof relies on knowing the effect of the intertwining integrals on the normalized Whittaker functional. Since the Whittaker functional is unique, the intertwining integral just multiplies it by a constant, which they computed.

In contrast with this algebraic case, Whittaker models of principal series representations of metaplectic groups are generally not unique. The effect of the intertwining operators on the Whittaker models was computed by Kazhdan and Patterson [26]. Specifically, they computed the scattering matrix of the intertwining operator corresponding to a simple reflection on the finite-dimensional vector space of Whittaker functionals for the $n$-fold metaplectic cover of $\mathrm{GL}(r, F)$, where $F$ is a $p$-adic field. Some terms in this matrix are simple rational functions of the Langlands parameters, while others involve $n$-th order Gauss sums. Though complicated in appearance, this scattering matrix was a key ingredient in their study of generalized theta series, and also in the later development of a metaplectic Casselman-Shalika formula by Chinta and Offen [16] and McNamara [35].

One of the two main results of this paper is that this scattering matrix computed by Kazhdan and Patterson is the $R$-matrix of a quantum group, quantum affine $\mathfrak{g l}(n)$, modified by Drinfeld twisting to introduce Gauss sums. This appears to be a new connection between

2010 Mathematics Subject Classification. Primary 16T25; Secondary 22E50. 
the representation theory of $p$-adic groups and quantum groups, which should allow one to use techniques from the theory of quantum groups to study metaplectic Whittaker functions.

Although we can now prove this directly, we were led to this result by studying lattice models whose partition functions give values of Whittaker functions on a metaplectic cover of $\operatorname{GL}(r, F)$. In [8], it was predicted that a solvable such model should exist; i.e., one for which a solution to the Yang-Baxter equation exists. Such a solvable model has important applications in number theory: it gives easy proofs (in the style of Kuperberg's proof of the alternating sign matrix conjecture) of several facts about Weyl group multiple Dirichlet series [11. The other main result of this paper is the discovery of a solvable lattice model whose partition function is a metaplectic Whittaker function. Moreover, we relate this solution to an $R$-matrix for the quantum affine superalgebra $\mathfrak{g l}(1 \mid n)$. The relation between the two main results follows from the inclusion of (quantum affine) $\mathfrak{g l}(n)$ into $\mathfrak{g l}(1 \mid n)$.

We now explain these results in more detail. Let $\widetilde{G}$ denote an $n$-fold metaplectic cover of $G:=\mathrm{GL}(r, F)$ where the non-archimedean local field $F$ contains the $2 n$-th roots of unity. Given a partition $\lambda$ of length $\leqslant r$, we will exhibit a system $\mathfrak{S}_{\lambda}$ whose partition function equals the value of one particular spherical Whittaker function at $\mathbf{s}\left(\operatorname{diag}\left(p^{\lambda_{1}}, \ldots, p^{\lambda_{r}}\right)\right)$, where $\mathbf{s}: \mathrm{GL}(r, F) \rightarrow \widetilde{G}$ is a standard section.

The systems proposed in [8] were generalizations of the six-vertex model. The six-vertex model with field-free boundary conditions was solved by Lieb [30], Sutherland [40] and Baxter [2] and were motivating examples that led to the discovery of quantum groups (cf. [29, 23, 17]). In Baxter's work, the solvability of the models is dictated by the Yang-Baxter equation where the relevant quantum group is $U_{q}\left(\widehat{\mathfrak{s l}}_{2}\right)$. In the special case $n=1$ (so when we are working with non-metaplectic $\mathrm{GL}(r, F)$ ), the systems proposed in [8] are six-vertex models that coincide with those discussed in Brubaker, Bump and Friedberg [9, 11] and there is a Yang-Baxter equation available. However even in this case these models differ from those considered by Lieb, Sutherland and Baxter since they are not field-free. Based on the results of this paper, we now understand that the relevant quantum group for the lattice models in [9, 11] is $U_{q}(\widehat{\mathfrak{g l}}(1 \mid 1))$, as we will make clear in subsequent sections.

It was explained in [8] that a Yang-Baxter equation for metaplectic ice would give new proofs of two important results in the theory of metaplectic Whittaker functions. The first is a set of local functional equations corresponding to the permutation of the Langlands-Satake parameters. The second is an equivalence of two explicit formulas for the Whittaker function, leading to analytic continuation and functional equations for associated Weyl group multiple Dirichlet series. The proof of this latter statement occupies the majority of [11].

However, no Yang-Baxter equation for the metaplectic ice in [8] could be found. In this paper we will make a small but crucial modification of the Boltzmann weights for the model in [8]. This change does not affect the partition function, but it makes possible a Yang-Baxter equation. This is Theorem 3.1 in Section 3. The solutions to the Yang-Baxter equation may be encoded in a matrix commonly referred to as an $R$-matrix.

We further prove that the resulting $R$-matrix has two important properties:

(1) It is a Drinfeld twist of the $R$-matrix obtained from the defining representation of quantum affine $\widehat{\mathfrak{g l}}(1 \mid n)$, a Lie superalgebra. 
(2) It contains the $R$-matrix of a Drinfeld twist of $\widehat{\mathfrak{g l}}(n)$ which, as we have already explained, we will identify with the scattering matrix of intertwining operators on Whittaker models for metaplectic principal series.

Consider the quantized enveloping algebra of the untwisted affine Lie algebra $\widehat{\mathfrak{g l}}(n)$, i.e. the central extension of the loop algebra of $\mathfrak{g l}(n)$. We denote the quantized enveloping algebra as $U_{\sqrt{v}}(\widehat{\mathfrak{g l} l}(n))$ instead of the usual $U_{q}$ because in our application the deformation parameter $v$ will be $q^{-1}$, where $q$ is the cardinality of the residue field of $F$. If $V$ and $W$ are vector spaces, let $\tau=\tau_{V, W}$ denote the flip operator $V \otimes W \rightarrow W \otimes V$. The Hopf algebra $U_{\sqrt{v}}(\widehat{\mathfrak{g l}}(n))$ is almost quasitriangular; given any two modules $V$ and $W$, there is an $R$-matrix $R_{V, W} \in \operatorname{End}(V \otimes W)$ such that $\tau R_{V, W}: V \otimes W \longrightarrow W \otimes V$ is a module homomorphism (though it will not always be an isomorphism). The $R$-matrices for $U_{\sqrt{v}}(\widehat{\mathfrak{g l} l}(n))$ acting on a tensor product of two evaluation modules were found by Jimbo [24] (see also Frenkel and Reshetikhin [19], Remark 4.1.); they satisfy a parametrized Yang-Baxter equation.

The quantum group $U_{\sqrt{v}}(\widehat{\mathfrak{g l}}(n))$ has an $n$-dimensional evaluation module $V_{+}(z)$ for every complex parameter value $z$. We will label a basis of the module $v_{+a}(z)$ where $a$ runs through the integers modulo $n$. The parameter $+a$ will be called a positive decorated spin (to be supplemented later by another one, denoted -0$)$. We may think of the decoration $a(\bmod$ $n)$ as roughly corresponding to the sheets of the metaplectic cover $\widetilde{G} \longrightarrow \operatorname{GL}(r)$ of degree $n$.

The resulting $R$-matrix in $\operatorname{End}\left(V_{+}\left(z_{1}\right) \otimes V_{+}\left(z_{2}\right)\right)$ is the matrix $R_{z_{1}, z_{2}}:=R_{V_{+}\left(z_{1}\right), V_{+}\left(z_{2}\right)}$ whose entries $R_{\alpha, \beta}^{\gamma, \delta}\left(z_{1}, z_{2}\right)$ are indexed by positive decorated spins $\alpha, \beta, \gamma$ and $\delta$ such that

$$
R_{z_{1}, z_{2}}\left(v_{\alpha}\left(z_{1}\right) \otimes v_{\beta}\left(z_{2}\right)\right)=\sum_{\gamma, \delta} R_{\alpha, \beta}^{\gamma, \delta}\left(z_{1}, z_{2}\right) v_{\gamma}\left(z_{1}\right) \otimes v_{\beta}\left(z_{2}\right) .
$$

These values are given by the following table:

\begin{tabular}{|c|c|c|c|}
\hline$\alpha, \beta, \gamma, \delta$ & $\begin{array}{c}+a,+a,+a,+a \\
\quad(0 \leqslant a \leqslant n)\end{array}$ & $\begin{array}{c}+b,+a,+b,+a \\
(0 \leqslant a, b \leqslant n, a \neq b)\end{array}$ & $\begin{array}{c}+b,+a,+a,+b \\
(0 \leqslant a, b \leqslant n, a \neq b)\end{array}$ \\
\hline$R_{\alpha, \beta}^{\gamma, \delta}\left(z_{1}, z_{2}\right)$ & $\frac{-v+\left(z_{1} / z_{2}\right)^{n}}{1-v\left(z_{1} / z_{2}\right)^{n}}$ & $g(a-b) \frac{1-\left(z_{1} / z_{2}\right)^{n}}{1-v\left(z_{1} / z_{2}\right)^{n}}$ & $\begin{cases}(1-v) \frac{\left(z_{1} / z_{2}\right)^{n}}{1-v\left(z_{1} / z_{2}\right)^{n}} & a>b, \\
(1-v) \frac{1}{1-v\left(z_{1} / z_{2}\right)^{n}} & a<b .\end{cases}$ \\
\hline
\end{tabular}

Here $g(a-b)$ is an $n$-th order Gauss sum. These are not present in the out-of-the-box $U_{\sqrt{v}}(\widehat{\mathfrak{g l}}(n)) R$-matrix, but may be introduced by Drinfeld twisting that will be discussed in Section 4 (see also Section 4 of [5]). This procedure does not affect the validity of the Yang-Baxter equations, but is needed for comparison with the $R$-matrix for the partition functions of metaplectic ice giving rise to Whittaker functions.

To obtain the full $R$-matrix used in the Yang-Baxter equation for metaplectic ice, we must enlarge the set of positive decorated spins $+a$ to include one more, labelled -0 . Thus there are $n+1$ decorated spins altogether, the positive ones and one more. The $n$-dimensional vector space $V_{+}(z)$ is enlarged to an $n+1$ "super" vector space $V_{ \pm}(z)$. The positive decorated spins are a basis for the odd part $V_{+}(z)$, and the even part $V_{-}(z)$ is one-dimensional, spanned by -0 . In Section 3, we present an $R$-matrix that gives a solution of the Yang-Baxter equation 
for the metaplectic ice model. In Section 4, we show that the solution of the Yang-Baxter equation is equivalent to the $R$-matrix corresponding to the defining representation of the quantum affine Lie superalgebra $U_{\sqrt{v}}(\widehat{\mathfrak{g l}}(1 \mid n))$ modified by a Drinfeld twist.

Finally, we explain the connection between the $R$-matrix of Theorem 3.1 and the structure constants alluded to in item (2) above. The local functional equations for metaplectic Whittaker functions mentioned earlier may be understood as arising from intertwining operators. Let $\widehat{T}$ be the diagonal torus in $\operatorname{GL}(r, \mathbb{C})$, the Langlands dual group of $G$. Each diagonal matrix

$$
\mathbf{z}=\left(\begin{array}{lll}
z_{1} & & \\
& \ddots & \\
& & z_{r}
\end{array}\right) \in \widehat{T}(\mathbb{C})
$$

indexes a principal series representation $\pi_{\mathbf{z}}$ of $\widetilde{G}$. Let $\mathcal{W}^{\mathbf{z}}$ be the finite-dimensional vector space of spherical Whittaker functions for $\pi_{\mathbf{z}}$. If $n=1, \mathcal{W}^{\mathbf{z}}$ is one-dimensional, but not in general since if $n>1$ the representation $\pi_{\mathbf{z}}$ does not have unique Whittaker models. If $s_{i}$ is a simple reflection in the Weyl group $W$, then let $\mathcal{A}_{s_{i}}$ denote the standard intertwining integral $\mathcal{A}_{s_{i}}: \pi_{\mathbf{z}} \longrightarrow \pi_{s_{i} \mathbf{z}}$ (see (5.5) for the precise definition). This induces a map $\mathcal{W}^{\mathbf{z}} \rightarrow \mathcal{W}^{s_{i} \mathbf{z}}$. If $n>1$ then $\mathcal{A}_{s_{i}}$ has an interesting scattering matrix on the Whittaker model that was computed by Kazhdan and Patterson (Lemma I.3.3 of [26]). This calculation underlies their work on generalized theta series, and was used by Chinta and Offen [16] and generalized by McNamara [35] to study the analog of the Casselman-Shalika formula for the spherical Whittaker functions.

Let $\pi_{\mathbf{z}, \psi}$ be the module of Whittaker coinvariants of the representation $\pi_{\mathbf{z}}$. By definition this is the quotient of the underlying space of $\pi_{\mathbf{z}}$ characterized by the fact that a linear functional is a Whittaker functional if and only if it factors through $\pi_{\mathbf{z}, \psi}$. Thus $\pi_{\mathbf{z}, \psi}$ is the dual space of the space of Whittaker functionals on $\pi_{\mathbf{z}}$. Its dimension is $n^{r}$. In Section 5 , we will prove that the scattering matrix of the intertwining integrals on the Whittaker coinvariants is essentially $\tau R_{z_{i}, z_{i+1}}$, where $R_{z_{i}, z_{i+1}}$ is the $R$-matrix for a Drinfeld twist of $U_{\sqrt{v}}(\widehat{\mathfrak{g l}}(n))$.

Theorem 1.1. There is an isomorphism $\theta_{\mathbf{z}}$ of the space $\pi_{\mathbf{z}, \psi}$ of Whittaker coinvariants to the vector space $V_{+}\left(z_{1}\right) \otimes \cdots \otimes V_{+}\left(z_{r}\right)$ that takes the vectors $v_{+a_{1}}\left(z_{1}\right) \otimes \cdots \otimes v_{+a_{r}}\left(z_{r}\right)$ into the basis of $\pi_{\mathbf{z}, \psi}$ dual to the basis of $\mathcal{W}^{\mathbf{z}}$ given in [26, 16, 35] (see Section 5). Then the following diagram commutes:

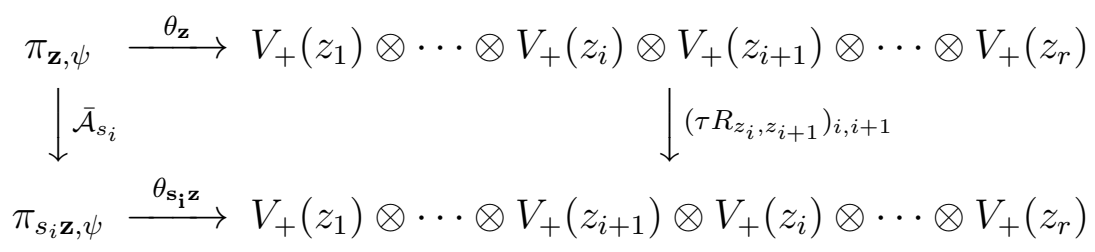

where $\overline{\mathcal{A}}_{s_{i}}$ denotes the map induced by the normalized intertwining operator defined in (5.6).

The notation $\left(\tau R_{z_{i}, z_{i+1}}\right)_{i, i+1}$ means that the operator $\tau R_{z_{i}, z_{i+1}}: V_{+}\left(z_{i}\right) \otimes V_{+}\left(z_{i+1}\right) \rightarrow$ $V_{+}\left(z_{i+1}\right) \otimes V_{+}\left(z_{i}\right)$ is applied to the $i, i+1$ tensor components, while we take the identity map on the remaining components. 
This offers a new and seemingly fundamental connection between the representation theory of quantum groups and $p$-adic metaplectic groups. It also suggests several immediate questions.

First, one may ask for generalizations to other Cartan types. For symplectic groups, YangBaxter equations based on those found here are given in Gray [22]. A categorical framework for some of these operations would be desirable. Even for central extensions of $\operatorname{GL}(r, F)$ there are open questions. We required the $2 n$-th roots of unity to be in the ground field $F$, in order to twist the Matsumoto cocycle defining the metaplectic central extension of $\operatorname{GL}(r, F)$ by a cocycle of the form $\left(\operatorname{det}\left(g_{1}\right), \operatorname{det}\left(g_{2}\right)\right)_{2 n}$ as in (5.1). We may ask whether other choices of cocycle admit a similar story; in particular, some choices result in a strictly smaller dimensional space of Whittaker models, so wouldn't biject with basis elements in the tensor product of vector spaces appearing in Theorem 1.1.

One may also ask for connections with other literature such as Weissman [41]. It seems particularly important to understand the relation between our work and the the quantum geometric Langlands program initiated by Lurie and Gaitsgory in [20], and more specifically the relation to the work of Lysenko [31] and Gaitsgory and Lysenko [21].

Remark 1.2. While we have given an interpretation of the $n+1$ decorated spins which are the possible states of the horizontal edges as basis vectors for an evaluation module of $U_{\sqrt{v}}(\widehat{\mathfrak{g l}}(1 \mid n))$, the edges of vertical type have no known similar interpretation. One may ask whether $U_{\sqrt{v}}(\widehat{\mathfrak{g l}}(1 \mid n))$ has a two-dimensional module $M$ such that the Boltzmann weights in Figure 2 are interpreted as the $R$-matrix for the pair $V_{z}, M$. We know no reason for such an $M$ to exist, except that if it does not, then Theorem 3.1 is an example of a parametrized Yang-Baxter equation that is not predicted by quasitriangularity.

We conclude by reviewing some recent papers which are sequels to this one.

The paper by Brubaker, Buciumas, Bump and Friedberg [5] was written after the first draft of this one was already posted to the arxiv, and depends on this one. In it we give a very general method of constructing representations of the affine Hecke algebra and show that examples of such representations can come either from the theory of Whittaker functionals on metaplectic $p$-adic groups or from certain Schur-Weyl dualities for quantum affine algebras. Theorem 1 in the present paper is used to prove the two representations mentioned are in fact the same. The paper also contains a more formal discussion of the Drinfeld twisting, an important supplement to the brief treatment we give below in Section 4 .

The paper by Brubaker, Buciumas, Bump and Gray [6] was also written after this one. It uses the Yang-Baxter equations from this paper, and supplementary ones from Gray [22], to reprove the main result of [11], which may be expressed as the equality of the partition functions of two different ice models. One of the two ice models is described below in Section 2, The other one is similar but has different weights. The equality of the two partition functions is reminiscent of dualities for physical systems, similar for example to the Kramers-Wannier duality that relates the partition functions of the low-temperature and high temperature Ising models.

In the paper Brubaker, Buciumas, Bump and Gustafsson [7] it is shown (extending the earlier paper [12] in the $n=1$ case) that the row transfer matrices for metaplectic ice can be interpreted as operators on the Fermionic Fock space $\mathfrak{F}$ of Kashiwara, Miwa and Stern [25], after Drinfeld twisting. This is a module for (twisted) $U_{\sqrt{v}}\left(\widehat{\mathfrak{s l}}_{n}\right)$. To achieve this one modifies 
the boundary conditions so that the grid has infinitely many columns. Then a sequence of spins in a row of vertically oriented edges may be interpreted as a basis vector in $\mathfrak{F}$, and the main theorem is that the row transfer matrices have expressions resembling vertex operators. In particular they are $U_{\sqrt{v}}\left(\widehat{\mathfrak{s l}}_{n}\right)$-module homomorphisms. This partially addresses the lack of an interpretation of the vertical edges as $U_{\sqrt{v}}\left(\widehat{\mathfrak{s l}}_{n}\right)$-modules noted in Remark 1.2.

Acknowledgements: This work was supported by NSF grants DMS-1406238 (Brubaker) and DMS-1001079, DMS-1601026 (Bump and Buciumas). We thank Gautam Chinta, Solomon Friedberg and Paul Gunnells for their support and encouragement, and David Kazhdan, Daniel Orr and the referee for helpful comments.

\section{THE PARTITION FUNCTION}

In statistical mechanics, the partition function of a model is a generating function. This means that through its dependence on global parameters of the system (such as temperature) it carries information about properties of the system such as entropy and free energy. Here we are concerned with two-dimensional lattice models that represent metaplectic Whittaker functions, and the global parameters on which it depends are the Langlands parameters.

Consider a finite two-dimensional rectangular grid of fixed size, composed of interior edges connecting to vertices of the grid and boundary edges adjacent to a single vertex in the grid. Every edge will be assigned a spin, which has value + or - . The spins along the boundary edges will be fixed as part of the data specifying the system; the spins on the interior edges will be allowed to vary. Thus with the spins on the boundary fixed, a state of the system will be an assignment of spins to the interior edges.

We associate a system to any integer partition $\lambda=\left(\lambda_{1}, \ldots, \lambda_{r}\right)$ as follows. The size of the grid will have $r$ rows and $N$ columns, where $N$ may be any integer greater than or equal to $\lambda_{1}+r$. The boundary edge spins are set to be + at all left and bottom boundary edges, and - on all right edges. The boundary edges along the top of the grid depend on the strict partition $\lambda+\rho$ with $\rho=(r-1, \ldots, 3,2,1,0)$. The spins along the top edge will be - in the columns numbered $(\lambda+\rho)_{i}$ for all $1 \leqslant i \leqslant r$ and + on all remaining columns. See Figure 1 for an example of a state in the system for $\lambda=(3,2,0)$ and $N=5.1$

Define the charge at each horizontal edge in the configuration to be the number of + spins at or to the right of the edge, along the same row. (This notion was introduced in [8].) We also will speak of the charge at a vertex, defined to be the charge on the edge to the right of the vertex. The charges are labeled in Figure 1 as decorations above each vertex.

Definition 2.1. The state will be called admissible if the four spins on adjacent edges of any vertex are in one of the six configurations in Figure 2 (top). It will be called $n$-admissible if it is admissible and if furthermore every horizontal edge with a - spin has charge $\equiv 0$ modulo $n$.

An example of an admissible state is shown in Figure 1. (The appearance of labels $z_{i}$ on the vertices in the figure will be explained momentarily.) The illustrated state is $n$-admissible only if $n=1$ or 2 , since it has a horizontal - edge with charge 2 .

\footnotetext{
${ }^{1}$ Strictly speaking, our systems correspond to an integer partition $\lambda$ and the choice of sufficiently large integer $N$ specifying the number of columns. However, the partition function $Z\left(\mathfrak{S}_{\lambda}\right)$ is unchanged if we increase $N$, and we suppress $N$ from the notation.
} 


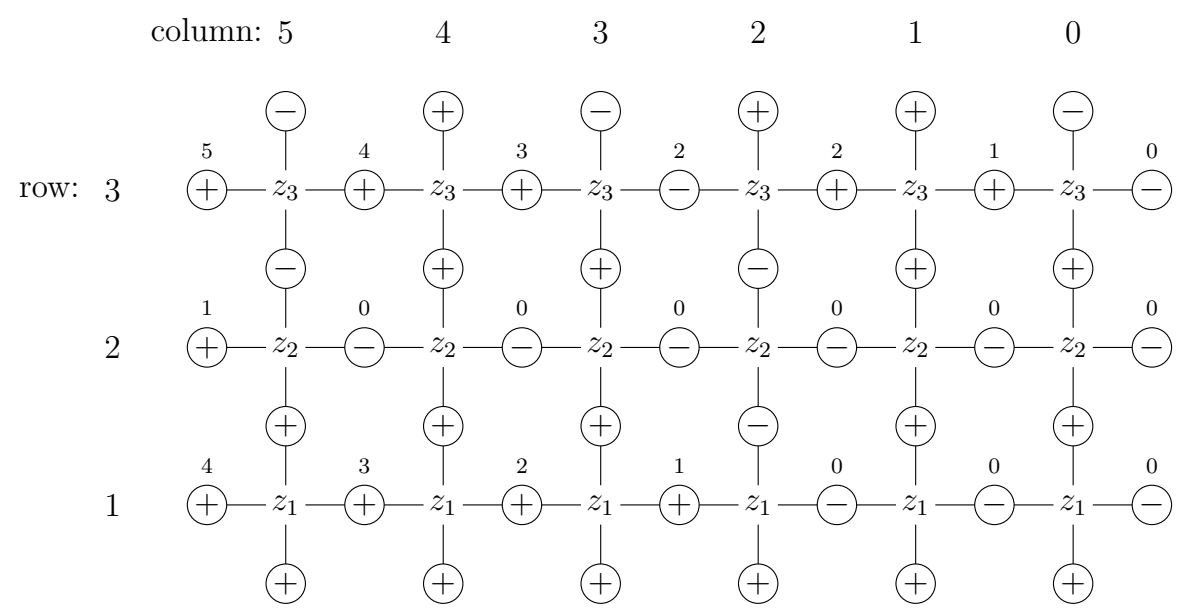

Figure 1. A state of a six-vertex model system. The columns are labeled in descending order from $N-1$ down to 0 . The rows are labeled in descending order from $r$ down to 1 . In this case $N=6$, the partition $\lambda$ is $(3,2,0)$, so $\lambda+\rho=(5,3,0)$; therefore the - in the top row are placed in columns 5,3 , 0 . The charges are shown for each horizontal edge. If $n=2$ this state is $n$-admissible since the charges of the - edges are multiples of 2 .

The Boltzmann weight of a state is obtained as a product of weights attached to each vertex in the model. The weight attached to any vertex makes use of a pair of functions $h$ and $g$ defined on the integers satisfying certain properties which we will now explain.

Let $n$ be a fixed positive integer and $v$ a fixed parameter. Let $g(a)$ be a function of the integer $a$ which is periodic modulo $n$, and such that $g(0)=-v$, while $g(a) g(n-a)=v$ if $n$ does not divide $a$. Let

$$
h(a)= \begin{cases}1-v & \text { if } n \mid a, \\ 0 & \text { otherwise. }\end{cases}
$$

Choose $r$ nonzero complex numbers $z_{1}, \ldots, z_{r}$ and associate one to each row, as indicated in Figure 1. The rows are labeled $r$ down to 1 in descending order and $z_{i}$ is associated with the $i$-th row as in Figure 1, Given a vertex in the $i$-th row, its Boltzmann weight is given in Figure 2 (top). Note that this weight depends on the spins and the charges on adjacent edges, and the row $i$ in which it appears. Then the Boltzmann weight $B_{z_{1}, \ldots, z_{r}}^{(n)}(\mathfrak{s})$ of the state $\mathfrak{s}$ is the product of the Boltzmann weights over all vertices in the grid. We often omit the $n$ or the $z_{1}, \ldots, z_{r}$ in the notation for $B$, as the weights may be stated uniformly for all such choices.

Remark 2.2. In [8] and [11], the functions $g$ and $h$ are defined using $n$-th order Gauss sums, with $v=q^{-1}$, and shown to satisfy the above properties. We will use this specific choice later in Theorem 2.5 and in Section 5 to connect the partition function to metaplectic Whittaker functions. However, only the above properties are required for their study using the Yang-Baxter equation. The function $g(a)$ is defined in (5.11) below, and $h(a)$ we already defined by (2.1).

Example 2.3. In the state in Figure 1 we look at the top row. Using the classification of admissible configurations in Figure 2 (top), the vertex in column 5 is of type $b_{1}$ with charge 


\begin{tabular}{|c|c|c|c|c|c|}
\hline$a_{1}$ & $\mathrm{a}_{2}$ & $b_{1}$ & $\mathrm{~b}_{2}$ & $C_{1}$ & $c_{2}$ \\
\hline$\underset{\oplus}{\oplus} \underset{1}{\oplus} \stackrel{a+1}{\oplus}$ & 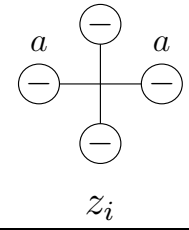 & 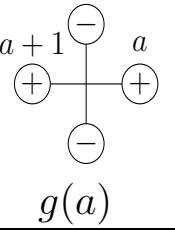 & 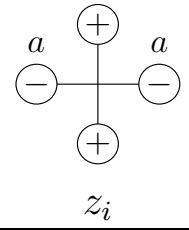 & 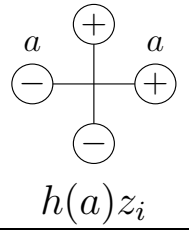 & $\stackrel{\oplus+{ }_{1}^{a+1}}{\Theta} \overbrace{1}^{a}$ \\
\hline 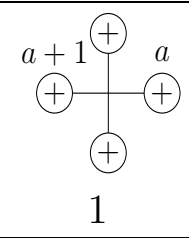 & $\stackrel{0}{-} \overbrace{-}^{-} \bigodot_{z_{i}}^{0}$ & 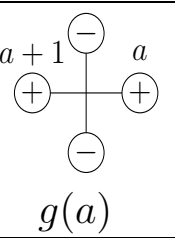 & 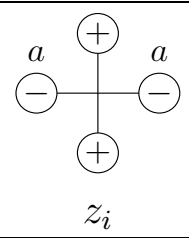 & 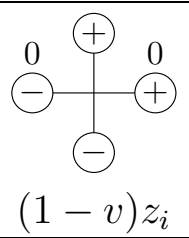 & 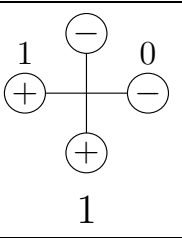 \\
\hline
\end{tabular}

Figure 2. The Boltzmann weights at a vertex. Top: the weights in Metaplectic Ice [8]. Bottom: the weights in this paper. These produce the same partition function but are subtly different in that the new weights satisfy a Yang-Baxter equation as in Theorem 3.1. The illustrated vertices are in the $i$-th row and have charge $a$. (The charge is the number of + signs in the row to the right of the vertex.) The Boltzmann weight of any configuration not appearing in this table is zero. In an $n$-admissible state, any horizontal edge with a - spin will have its charge divisible by $n$. Each admissible configuration is assigned a type $\mathrm{a}_{1}, \mathrm{a}_{2}, \mathrm{~b}_{1}, \mathrm{~b}_{2}, \mathrm{c}_{1}$ or $\mathrm{c}_{2}$.

4, so its Boltzmann weight is $g(4)$. There are two vertices of type $c_{2}$ in columns 3 and 0 , a $c_{1}$ vertex in column 2, and $\mathrm{a}_{1}$ vertices in columns 4 and 1 . The $c_{1}$ vertex and one of the $c_{2}$ vertices have charge 2 , so the state is $n$-admissible only if $n=1$ or 2 . Assuming this, the $g(4)$ from the $\mathrm{b}_{1}$ vertex evaluates to $-v$ and the $c_{1}$ vertex evaluates to $(1-v) z_{1}$, while the remaining vertices in the row have weight 1 . Thus the total contribution of this row to the weight of the state is $(-v)(1-v) z_{1}$. The second row has a vertex $c_{2}$ (with charge 0 ) and the remaining vertices are all of type $\mathrm{b}_{2}$ or $\mathrm{a}_{2}$, so this row contributes $z_{2}^{5}$. The last row has a $\mathrm{c}_{2}$ vertex (with charge 0 ) and two $b_{2}$ vertices. The remaining three vertices in the row are of type $\mathrm{a}_{1}$ with Boltzmann weight 1 . The Boltzmann weight of this state is $(-v)(1-v) z_{1} z_{2}^{5} z_{3}^{2}$ if $n=1$ or 2 , and 0 otherwise.

Proposition 2.4. Suppose that $\mathfrak{s}$ is an admissible state such that the Boltzmann weight $B^{(n)}(\mathfrak{s}) \neq 0$. Then the state is n-admissible.

Proof. We must show that, under this assumption, the charge on every edge with spin is a multiple of $n$. Suppose not and consider the right-most vertex in any row where this condition fails; that is, the charge $a$ of the edge to the right is not a multiple of $n$. We claim that the edge to the right of $v$ has spin + . We know that $v$ is not the rightmost vertex in its row since its charge is nonzero. So if the edge to the right of $v$ has spin - , then the vertex to the right of $v$ has the same charge as $v$, contradicting our assumption that $v$ is the rightmost counterexample in its row.

Since the edge to the left of $v$ is - and the edge to the right is + , consulting Figure 2 (top) we see that the only admissible configuration of spins at the vertex $v$ is of type $c_{1}$, so 
the Boltzmann weight at $v$ is $h(a) z_{i}=0$ because $n \nmid a$. This contradicts our assumption that $B^{(n)}(\mathfrak{s}) \neq 0$.

We may now explain the distinction between the system in [8] and the one used throughout this paper. Let $\mathfrak{S}_{\lambda}^{\prime}$ denote the set of admissible states, and let $\mathfrak{S}_{\lambda}$ denote the smaller set of $n$-admissible states. In [8], the partition function $Z\left(\mathfrak{S}_{\lambda}^{\prime}\right)$ is defined to be the sum of $B^{(n)}(\mathfrak{s})$ where $\mathfrak{s}$ runs over $\mathfrak{S}_{\lambda}^{\prime}$. In this paper, we consider partition function $Z\left(\mathfrak{S}_{\lambda}\right)$, the sum over $n$-admissible states.

Theorem 2.5. Let $\lambda$ be a partition with $r$ parts and let $n$ be a fixed positive integer. Then the partition function $Z\left(\mathfrak{S}_{\lambda}\right)$ is (up to normalization) a value of a p-adic spherical Whittaker function on the metaplectic n-fold cover of $\mathrm{GL}(r, F)$, where $F$ is a nonarchimedean local field with residue field of cardinality $q \equiv 1(2 n)$.

Proof. By Proposition [2.4, $Z\left(\mathfrak{S}_{\lambda}^{\prime}\right)=Z\left(\mathfrak{S}_{\lambda}\right)$. Combining this with Theorem 4 of [8], the statement follows.

In this result, $g(a)$ and $h(a)$ appearing in the Boltzmann weights for $Z$ are $n$-th order Gauss sums as explained in Remark 2.2 and $v=q^{-1}$ where $q$ is the cardinality of the residue field of $F$.

Remark 2.6. Theorem 4 of [8] depends on the crystal description of the Type A Whittaker functions proved in [33]. (See also [10].) However [33] treats simple groups such as $\operatorname{SL}(r)$. This apparent gap can be remedied by noting that the metaplectic Casselman-Shalika formula proved for reductive groups in [35] is equivalent to the needed crystal description by results of Puskas 38.

Remark 2.7. The normalization that is unspecified in this statement will be made precise in (6.2). Moreover, Theorem 6.3 is a generalization of this result that expresses a basis of all $n^{r}$ spherical Whittaker functions as partition functions of Gamma ice, and also elucidates the relationship with Theorem 1.1 .

To summarize, we may strictly limit the admissible states so that $-a$ only occurs with the charge $a \equiv 0$ modulo $n$. Thus we may use the Boltzmann weights of Figure 2 (bottom). The restriction to $n$-admissible states does not change the partition function but has the benefit of making the model solvable in the sense of Baxter. This means that it is amenable to study by the Yang-Baxter equation.

\section{The YAng-BaXter equation}

The partition functions described in Section 2 differ from those of the classical six-vertex model in a crucial way: the Boltzmann weights depend on a global statistic, the charge. If we wish to use statistical mechanical techniques like the Yang-Baxter equation, we need the weight at any vertex to be local, that is, depending only on nearest-neighbor interactions. We achieve this by a slight change in point of view, introducing decorated spins for the horizontal edges. Given a fixed positive integer $n$, a decorated spin is an ordered pair $(\sigma, a)$ where the spin $\sigma$ is + or - and the decoration $a$ is an integer $\bmod n$. Moreover if $\sigma=-$, we will only consider $a \equiv 0 \bmod n$. In figures we will sometimes draw the spin $\sigma$ in a circle and write the decoration $a$ next to it. In text we will denote $(\sigma, a)$ as $\sigma a$. The key point is that the decoration is now viewed as part of the data attached to a horizontal edge. Now there 
are $n+1$ possible decorated spins for horizontal edges, rather than just the spins + and - ; we have left the six-vertex model.

Not all choices of decorated spins on horizontal edges will have nonzero Boltzmann weight. Each decoration $a$ on a horizontal edge to the left of a vertex must be compatible with its spin $\sigma$ and the decoration $b$ on the edge to the right. If $\sigma=+$, then $a \equiv b+1(\bmod n)$ and if $\sigma=-$, then $a \equiv b$. If we set the initial decorations of the right-hand boundary edges (which all have spin -) to be 0 , then this rule clearly recovers the charge $(\bmod n)$ of the previous section. Thus the Boltzmann weights in Figure 2 may be interpreted as purely local; in the figure, we have indicated the decoration by writing it over the spin. We are justified in requiring the decoration at a - edge to be 0 modulo $n$ (without affecting the resulting partition functions) by Proposition 2.4.

Now that the weights at any vertex may be viewed as local, we are ready to present our solution to the Yang-Baxter equation. Three sets of vertices with different Boltzmann weights will appear in the Yang-Baxter equation (Theorem 3.1) below. In figures, these will be labeled $z_{i}, z_{j}$ and $R_{z_{i}, z_{j}}$. Here $z_{i}$ and $z_{j}$ are nonzero complex numbers used in the Boltzmann weight of the associated vertex. At the vertices with labels $z_{i}$ and $z_{j}$ we will use the Boltzmann weights already described in Figure 2 . In Figure 3 we describe the Boltzmann weights at the vertices labeled $R_{z_{i}, z_{j}}$. The Boltzmann weights depend only on the residue classes modulo $n$ of the integers $a, b, c, \ldots$ that appear in these formulas, but in some cases depend on a particular choice of representatives for residue classes. These choices are indicated in the description below the figure.

\begin{tabular}{|c|c|c|c|}
\hline 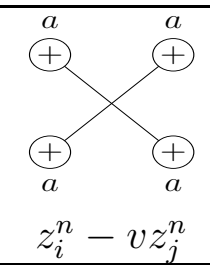 & $g(a-b)\left(z_{j}^{n}-z_{i}^{n}\right)$ & $(1-v) z_{j}^{c} z_{i}^{n-c}\left(^{*}\right)$ & $\overbrace{0}^{0} \overbrace{0}^{z_{j}^{n}-v z_{i}^{n}}$ \\
\hline$\stackrel{\oplus}{\oplus} \overbrace{a}^{0} \overbrace{0}^{a}$ & $\bigodot_{0}^{+} \overbrace{z_{i}^{n}-z_{i}^{n}}^{0}$ & $(1-v) z_{j}^{a} z_{i}^{n-a}(* *)$ & $\overbrace{(1-v)}^{0} \overbrace{a}^{n-a} z_{i}^{a}(* *)$ \\
\hline
\end{tabular}

Figure 3. Boltzmann weights for the $R$-vertex $R_{z_{i}, z_{j}}$. It is assumed that $b$ is not equal to $a .\left(^{*}\right)$ Here $c \equiv a-b \bmod n$ with $0 \leqslant c<n$. $\left({ }^{* *}\right)$ Here we choose the representative of $a$ modulo $n$ with $1 \leqslant a \leqslant n$, so if $a \equiv 0 \bmod n, n-a$ means 0 , not $n$. 
Theorem 3.1. The partition functions of the following two systems are equal. That is, if we fix the charges $\sigma, \tau, \beta, \rho, \alpha$ and $\theta$ and the decorations $a, b, c, d$, and sum over all possible values of the inner edge (decorated) spins, we obtain the same result in both cases.
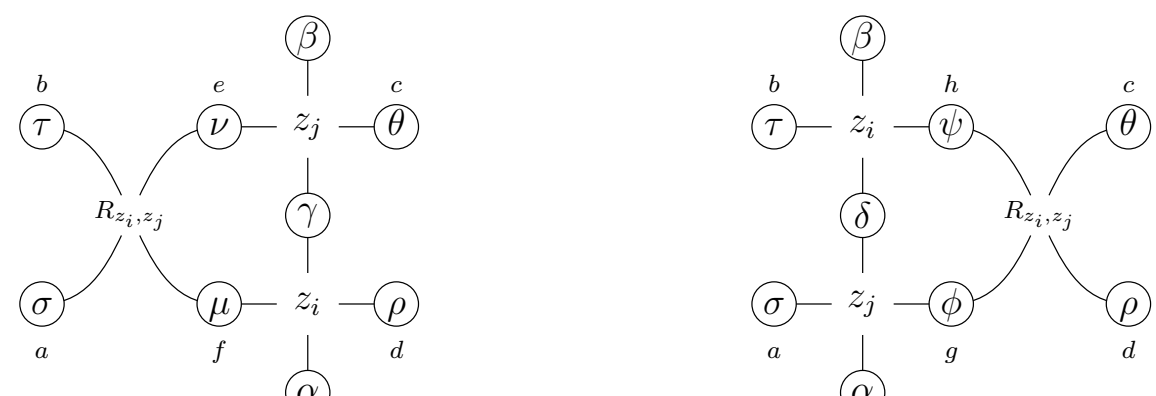

Proof. In every admissible configuration there are an even number of + spins on the six boundary edges. Therefore there are 32 possible boundary spin choices, and we must consider each of these cases separately. Moreover, each case breaks into subcases depending on the decorations at horizontal boundary edges with spins $\sigma, \tau, \theta$ and $\rho$. To give the reader a feeling for the possibilities, we will do one case in detail. The remaining cases may be found in [4].

We will consider Case 10, using the enumeration of cases in [4], whose assignment of boundary spins is $(\sigma, \tau, \beta, \theta, \rho, \alpha)=(+,+,-,+,-,+)$.

Case 10a: With $k \neq 0$, suppose that the (decorated) spins on the six boundary edges are as follows:

\begin{tabular}{|c|c|c|c|c|c|}
\hline$a$ & $b$ & & $c$ & $d$ & \\
$\sigma$ & $\tau$ & $\beta$ & $\theta$ & $\rho$ & $\alpha$ \\
\hline$k+1$ & 1 & & $k$ & 0 & \\
+ & + & - & + & - & + \\
\hline
\end{tabular}

On each side there is one $n$-admissible state:

left hand side

\begin{tabular}{|c|c|c|c|}
\hline$e$ & $f$ & & weight \\
$\nu$ & $\mu$ & $\gamma$ & \\
\hline$k+1$ & 1 & & $\left(z_{j}^{n}-z_{i}^{n}\right) g(k) g(-k)$ \\
+ & + & - & \\
\hline
\end{tabular}

Thus the configurations are as follows:

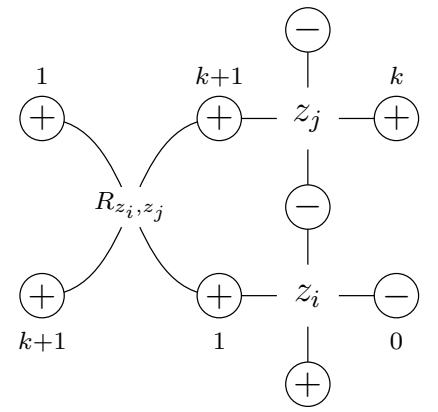

Since $g(k) g(-k)=v,(3.1)$ is satisfied in this case. 
Case 10b: With $k \neq 0$,

\begin{tabular}{|c|c|c|c|c|c|}
\hline$a$ & $b$ & & $c$ & $d$ & \\
$\sigma$ & $\tau$ & $\beta$ & $\theta$ & $\rho$ & $\alpha$ \\
\hline 1 & $k+1$ & & $k$ & 0 & \\
+ & + & - & + & - & + \\
\hline
\end{tabular}

left hand side

\begin{tabular}{|c|c|c|c|}
\hline$e$ & $f$ & & weight \\
$\nu$ & $\mu$ & $\gamma$ & \\
\hline $\begin{array}{c}k+1 \\
+\end{array}$ & 1 & & $(1-v) z_{j}^{k} z_{i}^{n-k} g(k)$ \\
\hline
\end{tabular}

right hand side

\begin{tabular}{|c|c|c|c|}
\hline$g$ & $h$ & & weight \\
$\phi$ & $\psi$ & $\delta$ & \\
\hline 0 & $k$ & & $(1-v) z_{j}^{k} z_{i}^{n-k} g(k)$ \\
- & + & - & \\
\hline
\end{tabular}

\section{Case 10c:}

\begin{tabular}{|c|c|c|c|c|c|}
\hline$a$ & $b$ & & $c$ & $d$ & \\
$\sigma$ & $\tau$ & $\beta$ & $\theta$ & $\rho$ & $\alpha$ \\
\hline 1 & 1 & & 0 & 0 & \\
+ & + & - & + & - & + \\
\hline
\end{tabular}

left hand side

\begin{tabular}{|c|c|c|c|}
\hline$e$ & $f$ & & weight \\
$\nu$ & $\mu$ & $\gamma$ & \\
\hline 1 & 1 & & $v\left(z_{j}^{n} v-z_{i}^{n}\right)$ \\
+ & + & - & \\
\hline
\end{tabular}

right hand side

\begin{tabular}{|c|c|c|c|}
\hline$g$ & $h$ & & weight \\
$\phi$ & $\psi$ & $\delta$ & \\
\hline 0 & 0 & & $(v-1) v z_{j}^{n}$ \\
- & + & - & \\
\hline 0 & 0 & & $\left(z_{j}^{n}-z_{i}^{n}\right) v$ \\
+ & - & + & \\
\hline
\end{tabular}

This exhausts all possible choices of decorations on boundary edges, and hence completes the proof of Case 10. See the appendix in [4] for the other cases.

There is another Yang-Baxter equation to be mentioned.

Theorem 3.2. Let $z_{i}, z_{j}$ and $z_{k}$ be given. Then for every choice of decorated boundary spins $\alpha, \beta, \gamma, \delta, \epsilon, \phi$, the partition functions of the following two systems are equal:
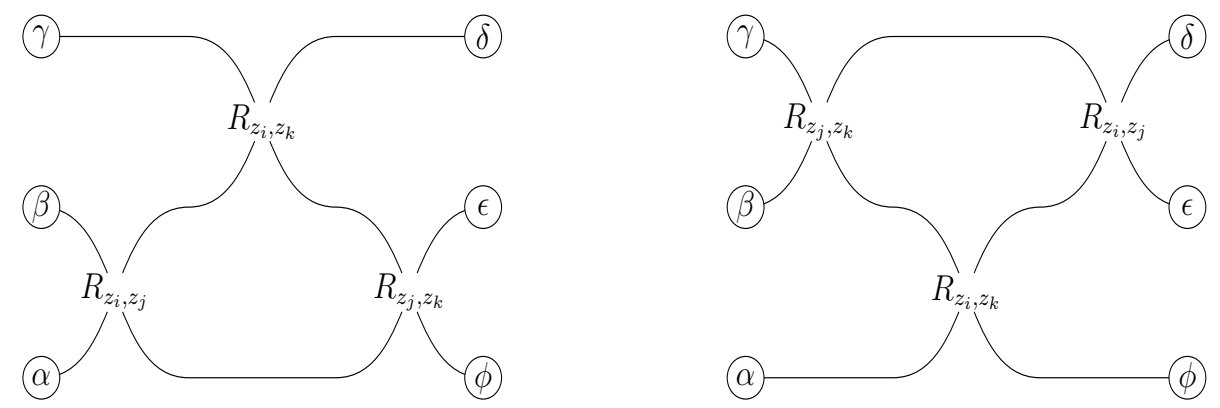

Theorem 3.2 presents a parametrized Yang-Baxter equation. We will eventually relate this to the parametrized Yang-Baxter equation associated with the $R$-matrix of a quantum group, namely a Drinfeld twist of $\widehat{\mathfrak{g l}}(1 \mid n)$. 
Proposition 3.3. Let $\alpha, \beta, \gamma, \delta$ be decorated spins. Then the partition function of

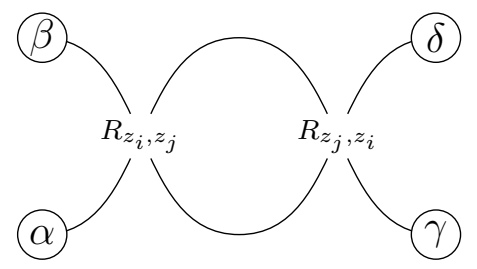

equals

$$
\begin{cases}\left(z_{j}^{n}-v z_{i}^{n}\right)\left(z_{i}^{n}-v z_{j}^{n}\right) & \text { if } \alpha=\gamma, \beta=\delta \\ 0 & \text { otherwise. }\end{cases}
$$

As we mentioned in the introduction, we will show in Section 5 that Theorem 3.2 is related to the intertwining integrals for principal series representations of the metaplectic group, which were calculated in Kazhdan and Patterson [26]. From this point of view, Proposition 3.3 is related to Theorem I.2.6 of [26].

Because of the last result, it is almost true that if we modified the $R$-matrix $R_{z_{i}, z_{j}}$ by dividing by $z_{j}^{n}-v z_{i}^{n}$, the associated quantum (super) group (which will be identified in Section (4) would be triangular in the sense of Drinfeld [17]. However because this factor $z_{j}^{n}-v z_{i}^{n}$ can be zero, this is not quite true, and the braided category of modules is also not triangular.

Proofs of Theorem 3.2 and Proposition 3.3. The earlier version [4] contains a proof that Theorem 3.2 and Proposition 3.3 follow from Theorem 3.1. However Theorem 3.2 may be proved more straightforwardly along the lines of Theorem 3.1 by consideration of the different cases, or deduced from Kojima [27] equation (2.13). We will verify at the end of the next section that Kojima's Yang-Baxter equation implies Theorem 3.2, and Proposition 3.3 is straightforward.

\section{Metaplectic Ice and Supersymmetry}

Perk and Schultz [37] found new solutions of the Yang-Baxter equation. Meanwhile graded (supersymmetric) Yang-Baxter equations were introduced by Bazhanov and Shadrikov [3]. It was found by Yamane [42] that the Perk-Schultz equations were related to the $R$-matrix of the quantized enveloping algebra of the $\mathfrak{g l}(m \mid n)$ Lie superalgebra in the standard representation. The quantized enveloping algebra of the corresponding affine Lie superalgebra was considered by Zhang [44]. A convenient reference for us is Kojima [27. See also [43].

We will explain how to relate the $R$-vertex weights of the prior section, which depend on a fixed $n$ as in Figure 3, to the $\mathfrak{g l}(1 \mid n) R$-matrix. The relationship is rather subtle, since we will have to perform manipulations on the Perk-Schultz $R$-matrix in order to make the comparison. These manipulations preserve the Yang-Baxter equation as in Theorem 3.2, but (among other things) they introduce $n$-th order Gauss sums which are crucial in the connection to representation theory of the metaplectic group [26, 8, 11].

If $V$ is the $(1 \mid n)$-dimensional defining module of quantum $\mathfrak{g l}(1 \mid n)$, then for every $z \in \mathbb{C}^{\times}$ there is an evaluation module $V_{z}$ of $U_{q}(\widehat{\mathfrak{g l}}(1 \mid n))$. One can associate $R$-matrices $R_{z_{i}, z_{j}} \in$ $\operatorname{End}\left(V_{z_{i}} \otimes V_{z_{j}}\right)$ (see [27] for more details) that satisfy a graded Yang-Baxter equation in $\operatorname{End}\left(V_{z_{i}} \otimes V_{z_{j}} \otimes V_{z_{k}}\right)$. As noted in [27], we may change some signs in the $R$-matrix to produce a solution to the ungraded Yang-Baxter equation; this is the $R$-matrix we wish to compare 
with that in Theorem 3.2. A basis of the ungraded $(1 \mid n)$-dimensional vector space $V_{z}$ can be taken to be the decorated edge spins -0 for the even part, and $+a$ with $a$ modulo $n$ for the odd part, of a vertex with parameter $z$.

Referring to [27] for notation, we will take the decorated spin -0 to have graded degree 0 , and the spins $+a$, where $a$ is an integer modulo $n$, to have degree 1 . Thus we are concerned with $\widehat{\mathfrak{g l}}(1 \mid n)$. For the sake of comparing our results to Kojima's, the parameter $q$ in this section will be Kojima's $q$, which will equal $\sqrt{v}$; it is not the same as $q$ (the cardinality of the $p$-adic residue field) in the other sections of this paper.

In Figure 4 we have the Boltzmann weights from Figure 3 divided by $z_{i}^{n}$, compared with the corresponding $R$-matrix entries from (2.4)-(2.7) of [27] which we have multiplied by the constant $1-q^{2} z$.

We will give two ways of modifying the $R$-matrix to obtain another $R$-matrix that is also a solution of the Yang-Baxter equation. One method only affects the weights in cases III, VII and VIII. The other only affects the weights in cases II, V and VI. After these changes, we will be able to match the Kojima Boltzmann weights up to sign, with $z=z_{i} / z_{j}$ and $q^{2}=v$. (Then we will have to discuss the sign.)

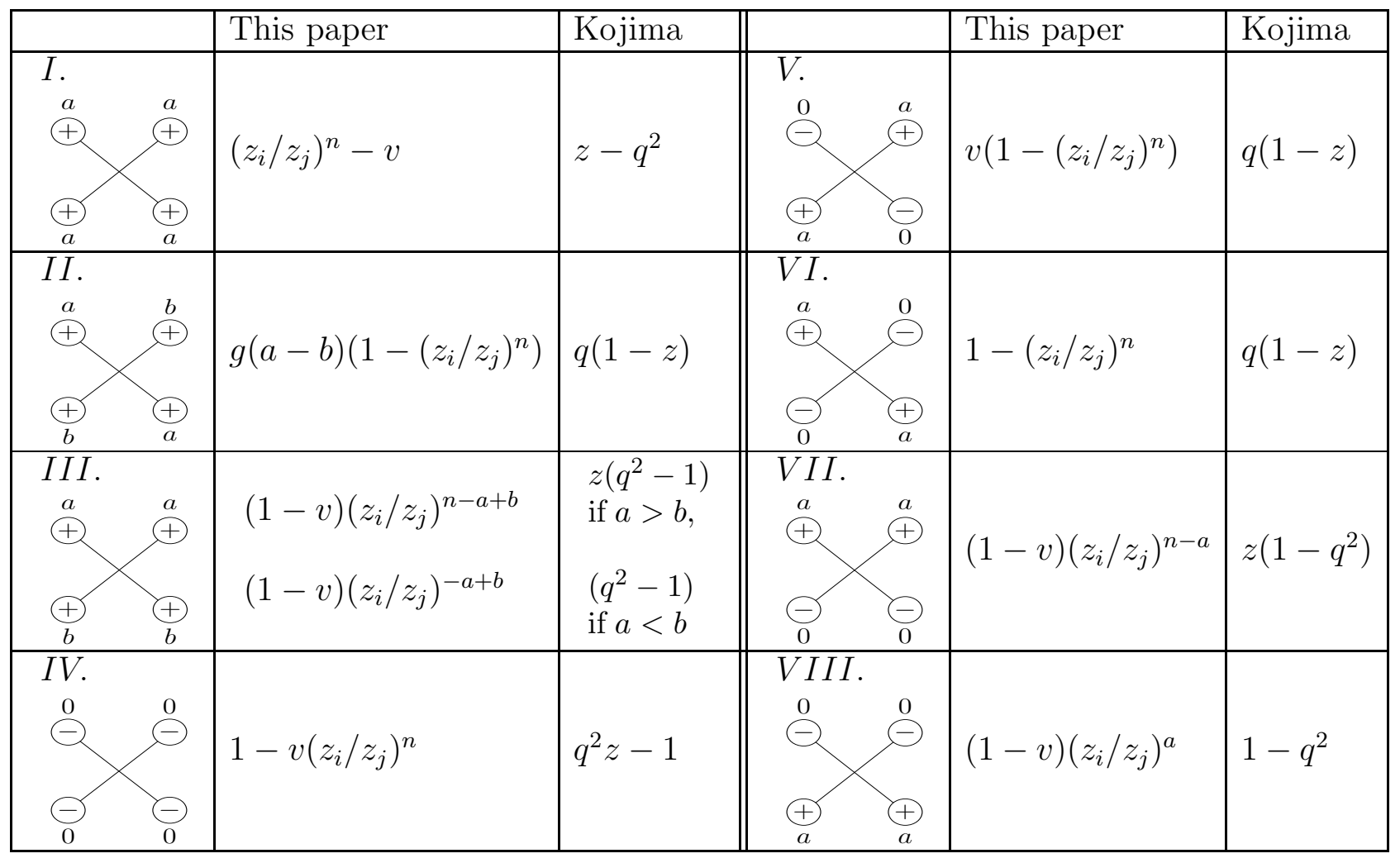

Figure 4. Left: The $R$-matrix from Figure 3, divided by $z_{j}^{n}$. Right: the Boltzmann weights of Kojima's $\widehat{\mathfrak{g l}}(1 \mid n) R$-matrix multiplied by $1-q^{2} z$. Just taking the first three cases (and discarding any case with a decorated spin -0) gives the $\widehat{\mathfrak{g l}}(n) R$-matrix. It is assumed that $a \not \equiv b \bmod n$. 
For each nonzero complex number $z$, let $V(z)$ be an $(n+1)$-dimensional vector space with basis $v_{\alpha}=v_{\alpha}(z)$, where $\alpha$ runs through $n+1$ "decorated spins." These are the ordered pairs $+a$ with $0 \leqslant a<n$ and -0 .

Previously we interpreted the vertex $R_{z_{i}, z_{j}}$ as a vertex in a graph with certain Boltzmann weights attached to it. We now reinterpret it as an endomorphism of a vector space, as usual in the application of quantum groups to solvable lattice models. If $\alpha, \beta, \gamma, \delta$ are decorated spins, let $R_{\alpha, \beta}^{\gamma, \delta}\left(z_{i}, z_{j}\right)$ be the Boltzmann weight of vertex $R_{z_{i}, z_{j}}$ with the decorated spins $\alpha, \beta, \gamma, \delta$ arranged as follows:

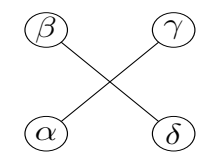

We assemble these into an endomorphism $R_{z_{i}, z_{j}}$ of $V\left(z_{1}\right) \otimes V\left(z_{2}\right)$ as follows:

$$
R_{z_{i}, z_{j}}\left(v_{\alpha} \otimes v_{\beta}\right)=\sum_{\gamma, \delta} R_{\alpha, \beta}^{\gamma, \delta}\left(z_{i}, z_{j}\right) v_{\gamma} \otimes v_{\delta}
$$

Lemma 4.1. We have

$$
\left(R_{z_{j}, z_{k}}\right)_{23}\left(R_{z_{i}, z_{k}}\right)_{13}\left(R_{z_{i}, z_{j}}\right)_{12}=\left(R_{z_{i}, z_{j}}\right)_{12}\left(R_{z_{i}, z_{k}}\right)_{13}\left(R_{z_{j}, z_{k}}\right)_{23}
$$

as endomorphisms of $V\left(z_{1}\right) \otimes V\left(z_{2}\right) \otimes V\left(z_{3}\right)$.

Here the notation is (as usual in quantum group theory) that $X_{i j}$ where $1 \leqslant i<j \leqslant 3$ means a matrix $X$ acting on the $i, j$ components in $V\left(z_{1}\right) \otimes V\left(z_{2}\right) \otimes V\left(z_{3}\right)$ with the identity acting on the third component.

Proof. We apply the left-hand side of (4.2) to $v_{\alpha} \otimes v_{\beta} \otimes v_{\gamma}$ and extract the coefficient of $v_{\delta} \otimes v_{\varepsilon} \otimes v_{\phi}$. This is found to be

$$
\sum_{\mu, \nu, \sigma} R_{\alpha, \beta}^{\mu, \sigma}\left(z_{i}, z_{j}\right) R_{\mu, \gamma}^{\delta, \nu}\left(z_{i}, z_{k}\right) R_{\sigma, \nu}^{\varepsilon, \phi}\left(z_{j}, z_{k}\right)
$$

which is the partition function of the first system in Theorem 3.2. The same calculation applied to the right hand side of (4.2) gives the partition function of the second system in Theorem 3.2. So they are equal.

We will now describe two operations that one may perform on the Boltzmann weights that do not affect the validity of the Yang-Baxter equation.

Change of basis. We may change basis in $V(z)$. Let $f(\alpha, z)$ be a function of a decorated spin $\alpha$ and a complex number $z$. Let $u_{\alpha}=f(\alpha, z) v_{\alpha}$ for $v_{\alpha} \in V(z)$. Then

$$
R_{z_{i}, z_{j}}\left(u_{\alpha} \otimes u_{\beta}\right)=\sum_{\gamma, \delta} \hat{R}_{\alpha, \beta}^{\gamma, \delta}\left(z_{i}, z_{j}\right) u_{\gamma} \otimes u_{\delta}
$$

where

$$
\hat{R}_{\alpha, \beta}^{\gamma, \delta}=\frac{f\left(\alpha, z_{i}\right) f\left(\beta, z_{j}\right)}{f\left(\gamma, z_{i}\right) f\left(\delta, z_{j}\right)} R_{\alpha, \beta}^{\gamma \delta} .
$$

Note that replacing $R$ by $\hat{R}$ only affects the weights in cases III, VII, and VIII in Figure 4 . 
Let us translate this into the language of Boltzmann weights. At the moment we are only concerned with Theorem 3.2. Later in Section 5 we will apply this technique to the first Yang-Baxter equation in Theorem 3.1. Thus we note the effect on the weights for both types of vertices. Taking the Boltzmann weights from Figures 3 and 2, with $z_{i}$ and $z_{j}$ as in those figures, the weights of
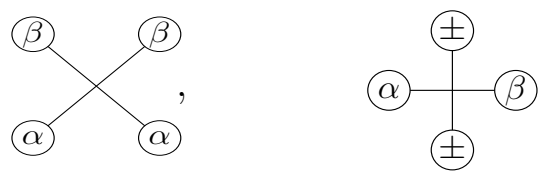

will respectively be multiplied by

$$
\frac{f\left(\alpha, z_{i}\right) f\left(\beta, z_{j}\right)}{f\left(\gamma, z_{i}\right) f\left(\delta, z_{j}\right)}, \quad \frac{f\left(\alpha, z_{i}\right)}{f\left(\beta, z_{i}\right)} .
$$

The first statement is a paraphrase of (4.3), and the second is checked the same way.

Returning to the comparison with Kojima's weights, we take

$$
f(\alpha, z)= \begin{cases}z^{a}, & \alpha=+a, \\ 1 & \alpha=-0 .\end{cases}
$$

This puts our $R$-matrix into agreement with Kojima in cases III, VII, and VIII but has no effect on the other cases. The modification in this subsection did not fundamentally change the $R$-matrix, or the quantum group associated to it. We simply made a change of basis in the vector space on which it acts.

Twisting. In this subsection we will consider a more fundamental change of the $R$-matrix which does not affect the validity of the Yang-Baxter equation. This procedure is called Drinfeld twisting [18]. The quantum group associated to the twisted $R$-matrix is not the original quantum group, as the Drinfeld twisting procedure modifies the comultiplication and universal $R$-matrix of a quasitriangular Hopf algebra. See Chari and Pressley [15] Section 4.2.E for more details. In Reshetikhin [39] Section 3, Drinfeld twisting is used to obtain multiparameter deformations of $U_{q}(\mathfrak{s l}(n))$. We explain in [5], Section 4 (at least for the $\mathfrak{g l}(n)$ part) how the Drinfeld twist on the quantum group produces the desired change to the $R$-matrix that we present below.

Notice that in Figure 3, if we have a nonzero weight for the vertex of form

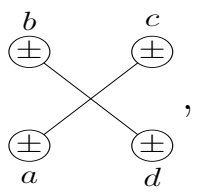

then either $a=c$ and $b=d$ or $a=d$ and $b=c$.

Now let us consider a modification of the Boltzmann weights in case II (i.e., $a=c$ and $b=d)$. We will multiply this weight by a function $\phi(a, b)$ of the decorations $a, b$ that has the following properties. First, it is independent of $z_{i}$ and $z_{j}$. Second, $\phi(a, b) \phi(b, a)=1$.

Proposition 4.2. If $\widetilde{R}$ is the R-matrix with this modification of the weights in case II, then $\widetilde{R}$ also satisfies the same Yang-Baxter equation that $R$ does (Theorem 3.2 ). 
Proof. From the Boltzmann weights in Figure 4, we see that the decorated spins of the two edges to the right of the vertex will have the same decorations as the two edges to the left of the vertex, in some order. From the form of $R_{z_{i}, z_{j}}$ it is clear that if either partition function is nonzero, the decorated spins $\delta, \epsilon$ and $\phi$ must be the same as $\alpha, \beta$ and $\gamma$ in some order. From this ordering, we may infer the number of case II vertices, and (with an exception to be explained below) it will be the same for both partition functions. That is, if $+a$ and $+b$ occur on the left in the opposite order that they do on the right, then a case II crossing must occur somewhere on a vertex between the four edges. And this will be true on both sides of the equation, so multiplying the case II Boltzmann weight by $\phi(a, b)$ will have the same effect on both sides of the equation.

The exception is that if two weights appear in the same order on the left and right, there may be two case II vertices or none between them. Thus suppose that $\alpha=\phi=+a$ and $\beta=\epsilon=+b$. Then in the first partition function in Proposition 3.3 we may have $R_{z_{i}, z_{j}}$ and $R_{z_{j}, z_{k}}$ either both in case II or both in case III. However if they are both in case II, the factor that we have to multiply is $\phi(a, b) \phi(b, a)$, which equals 1 by assumption.

We may use this method of twisting in order to remove the $g(a-b)$ in case II, and replace them by $q$, since in this case $a \not \equiv b \bmod n$, so $g(a-b) g(b-a)=v=q^{2}$. We may also adjust the weights in cases V and VI so that in both cases the coefficient agrees with Kojima's weights.

Sign. Using the two methods available to us, we see that we can adjust the Boltzmann weights to agree with Kojima's, up to sign. We must now discuss the sign. We have agreement for all signs except case IV. As Kojima notes (below his equation (2.12)) his $R$ matrix, being supersymmetric, satisfies a graded Yang-Baxter equation. As he points out, an ungraded Yang-Baxter equation may be obtained by changing the sign when all edges are odd-graded. For us, this would mean changing the sign in cases I, II and III. However it works equally well to change the sign in the case where all edges are even-graded, that is, in case IV.

In conclusion, putting together the results of all of the above subsections, the supersymmetric Yang-Baxter equation in Kojima [27] is equivalent to our Theorem 3.2.

\section{INTERTWINING INTEGRALS AS $R$-MATRICES}

In this section, we will review results of Kazhdan and Patterson [26], Chinta and Offen [16] and McNamara [34, 35] concerning the scattering matrix of the intertwining operators of the principal series representations on their Whittaker models. Then we return to the $R$ matrices, using modified Boltzmann weights that are suited to make a connection with the notation of [35], which will be our primary reference. Finally we will prove Theorem 1.1.

Let $F$ be a non-archimedean local field with ring of integers $\mathfrak{o}$ and a choice of local uniformizer $\varpi$. Let $q$ be the cardinality of the residue field $\mathfrak{o} / \varpi \mathfrak{o}$. Let $n$ be a fixed positive integer. We assume that $q \equiv 1(\bmod 2 n)$ so that $F$ contains the $2 n$-th roots of unity. Let $\mu_{n}$ denote the group of $n$-th roots of unity in $F$ and fix an embedding $\mu_{n} \longrightarrow \mathbb{C}^{\times}$.

Let $G:=\mathrm{GL}(r, F)$ and let $T$ be the subgroup of diagonal matrices. We begin by con-

structing a metaplectic $n$-fold cover of $G$, denoted $\tilde{G}^{(n)}$ or just $\tilde{G}$ when the degree of the 
cover is understood. Recall that $\tilde{G}$ is constructed as a central extension of $G$ by $\mu_{n}$ :

$$
1 \longrightarrow \mu_{n} \longrightarrow \tilde{G} \stackrel{p}{\longrightarrow} G \longrightarrow 1 \text {. }
$$

Thus as a set, $\tilde{G} \simeq G \times \mu_{n}$, but the multiplication in $\tilde{G}$ is dictated by a choice of cocycle $\sigma$ for $H^{2}\left(G, \mu_{n}\right)$. One may construct the cocycle explicitly, as in Kubota [28], Matsumoto [32, Kazhdan and Patterson [26] and Banks-Levi-Sepanski [1], or realize the central extension as coming from an extension of $K_{2}(F)$ constructed by Brylinski-Deligne [13]. For the applications at hand, we need only a few facts about the multiplication on $\tilde{T}=p^{-1}(T)$, the inverse image of a maximal split torus $T$ in $G$, and the splitting properties of some familiar subgroups.

Our cocycle $\sigma$ is chosen so that its restriction to $T \times T \longrightarrow \mu_{n}$ is given on any $\mathbf{x}, \mathbf{y}$ in $T$ explicitly by

$$
\sigma(\mathbf{x}, \mathbf{y})=\sigma\left(\left(\begin{array}{ccc}
x_{1} & & \\
& \ddots & \\
& & x_{r}
\end{array}\right),\left(\begin{array}{ccc}
y_{1} & & \\
& \ddots & \\
& & y_{r}
\end{array}\right)\right)=(\operatorname{det}(\mathbf{x}), \operatorname{det}(\mathbf{y}))_{2 n} \prod_{i>j}\left(x_{i}, y_{j}\right)^{-1},
$$

where $(\cdot, \cdot): F^{\times} \times F^{\times} \longrightarrow \mu_{n}$ is the $n$-th power Hilbert symbol and $(\cdot, \cdot)_{2 n}$ is the $2 n$-th power Hilbert symbol, so $(x, y)=(x, y)_{2 n}^{2}$. General properties of the Hilbert symbol may be found in [36] noting that the symbol there is the inverse of ours; one property we use frequently is that $(x, x)=1$ for any element $x \in F^{\times}$, since $F$ contains the $2 n$-th roots of unity.

Let $\Lambda=X_{*}(T)$ denote the group of rational cocharacters of $T$. The cocycle $\sigma$ in (5.1) is the inverse of the one appearing on p. 39 of [26]. A short computation shows that the commutator of any pair of elements $\tilde{\mathbf{x}}, \tilde{\mathbf{y}}$ in $\tilde{T}$ projecting to $\mathbf{x}$ and $\mathbf{y}$, respectively, in $T$ is

$$
[\tilde{\mathbf{x}}, \tilde{\mathbf{y}}]=\prod_{i=1}^{r}\left(x_{i}, y_{i}\right)
$$

In particular if $x, y \in F^{\times}$and $\lambda, \mu$ are elements of $X_{*}(T)$, let $\widetilde{\lambda(x)}, \widetilde{\mu(y)} \in \tilde{T}$ map to $x^{\lambda}$ and $y^{\mu}$, respectively, under the projection $p$ to $T$. Then according to (5.2),

$$
[\widetilde{\lambda(x)}, \widetilde{\mu(y)}]=(x, y)^{\langle\lambda, \mu\rangle}
$$

where $\langle\cdot, \cdot\rangle$ denotes the usual dot product on $X_{*}(T) \simeq \mathbb{Z}^{r}$.

In order to make use of results in [35], we must connect this explicit construction to the one used there. In [35] the construction of $\tilde{G}$ is obtained by first constructing the extension of $G(F)$ by $K_{2}(F)$ using a $W$-invariant quadratic form $Q$, and then using a push forward from $K_{2}(F)$ to the residue field, containing $\mu_{n}$. The calculation in (5.3) implies that the bilinear form $B(\lambda, \mu):=Q(\lambda+\mu)-Q(\lambda)-Q(\mu)$ for our extension, as described in Equation (2.1) of [35], is given by the dot product. If $\alpha$ is a (co)root then $Q(\alpha)=1$.

Finally, we record that the cocycle splits over any unipotent subgroup and over the maximal compact subgroup $K=\operatorname{GL}(r, \mathfrak{o})$ has a splitting in $\tilde{G}$. The splitting over the maximal unipotent is clear from the description of the cocycle in [26] and the splitting over $K$ is their Proposition 0.1.2. By abuse of notation, we will denote the image of $K$ in $\tilde{G}$ also as $K$.

Let $T(\mathfrak{o})=K \cap T$, and let $\tilde{T}(\mathfrak{o})$ be the preimage of $T(\mathfrak{o})$ in $\widetilde{G}$. Let $H$ be the centralizer of $\tilde{T}(\mathfrak{o})$ in $\tilde{T}$. It consists of elements in $\tilde{T}$ whose projection to the torus $\mathbf{t}=\left(t_{1}, \ldots, t_{r}\right) \in$ $T \simeq\left(F^{\times}\right)^{r}$ has $\operatorname{ord}_{\varpi}\left(t_{j}\right) \equiv 0(n)$ for $j=1, \ldots, r$. The subgroup $H$ is abelian. Thus we may 
identify $\tilde{T} / \mu_{n} \tilde{T}(\mathfrak{o})$ and $H / \mu_{n} \tilde{T}(\mathfrak{o})$ with lattices $\Lambda$ and $n \Lambda$, respectively. In particular $\Lambda$ is isomorphic to the cocharacter lattice $X_{*}(T)$ of $T$. The map $\lambda \mapsto \varpi^{\lambda}$ induces an isomorphism from $X_{*}(T)$ to $T / T(\mathfrak{o})$. Let $\mathbf{s}: G \rightarrow \tilde{G}$ denote the standard section. By abuse of notation we will also denote by $\varpi^{\lambda}$ the image of $\varpi^{\lambda}$ under s. Let $\rho=(r-1, \ldots, 2,1,0)$ and let $\Gamma$ be the set of $\nu \in \Lambda=\mathbb{Z}^{r}=X_{*}(T)$ such that

$$
\nu-\rho=\left(c_{1}, \ldots, c_{r}\right), \quad \text { with } c_{i} \in\{0, \ldots, n-1\} \text { for all } i .
$$

This is a set of coset representatives in $\Lambda=\mathbb{Z}^{r}=X_{*}(T)$ for $\Lambda$ modulo $n \Lambda$. Then $\left\{\varpi^{\lambda} \mid \lambda \in \Gamma\right\}$ are a set of coset representatives for $\tilde{T} / H$.

Next we recall the construction of the genuine unramified principal series on $\tilde{G}$. (A representation $\pi$ of $\tilde{G}$ or any subgroup containing $\mu_{n}$ is called genuine if $\pi(\varepsilon g)=\varepsilon \pi(g)$ for $\varepsilon \in \mu_{n}$, where we are using the fixed embedding of $\mu_{n} \subset F^{\times}$into $\mathbb{C}^{\times}$.) First we construct geniune irreducible representations of $\tilde{T}$. Let $\chi$ be a genuine character of $H$ that is trivial on $\tilde{T} \cap K$; the induced representation $i(\chi)$ of such a character to $H$ will be irreducible.

Now we may parabolically induce $i(\chi)$ to $\tilde{G}$. This is done by first inflating the representation from $\tilde{T}$ to $\tilde{B}$, the inverse image of the standard Borel subgroup $B \supset T$ in $G$ and then inducing to obtain $I(\chi):=\operatorname{Ind}_{\tilde{B}}^{\tilde{G}}(i(\chi))$. Explicitly $I(\chi)$ is the space of locally constant functions $f: \tilde{G} \longrightarrow i(\chi)$ such that

$$
f(b g)=\delta^{1 / 2} \chi(b) f(g) \text { for all } g \in \tilde{G}, b \in \tilde{B},
$$

where $\delta$ denotes the modular quasicharacter of $B$. Thus $I(\chi)$ is a $\tilde{G}$-module under the action of right translation. Let $\phi_{K}:=\phi_{K}^{\chi}$ denote any of the $i(\chi)$-valued functions in the one-dimensional space of $K$-fixed vectors in $I(\chi)$; our results will be independent of this choice.

The characters $\chi$ of $H$ that are trivial on $\tilde{T} \cap K$ may be parametrized by elements $\mathbf{z} \in \widehat{T}$, which is the group of diagonal elements of $\mathrm{GL}(r, \mathbb{C})$. Every element of $H$ may be written $\varepsilon \mathbf{s}(t)$ with $\varepsilon \in \mu_{n}$ and $t=\operatorname{diag}\left(t_{1}, \cdots, t_{r}\right) \in T$ such that each $\operatorname{ord}\left(t_{i}\right)$ is a multiple of $n$. We may then define

$$
\chi_{\mathbf{z}}(\varepsilon \mathbf{s}(t))=\varepsilon \prod_{i=1}^{r} z_{i}^{\operatorname{ord}\left(t_{i}\right)} .
$$

We will denote the corresponding principal series representation $\pi_{\mathbf{z}}=I\left(\chi_{\mathbf{z}}\right)$. It does not depend uniquely on $\mathbf{z}$ since if $\mathbf{z}^{n}=\left(\mathbf{z}^{\prime}\right)^{n}$ then $\pi_{\mathbf{z}} \cong \pi_{\mathbf{z}^{\prime}}$.

We will assume that $I\left(\chi_{\mathbf{z}}\right)$ is irreducible. For this it is necessary and sufficient to assume that $\mathbf{z}^{n \alpha} \neq q^{ \pm 1}$ for all roots $\alpha$. This also guarantees that the rational functions that appear in the sequel do not have poles.

Let $U$ be the subgroup of upper unitriangular matrices in $G$, which is the unipotent radical of $B$, the positive Borel subgroup. The Matsumoto construction supplies a splitting of the metaplectic cover over $U$, so by abuse of notation we may regard $U$ as a subgroup of $\tilde{G}$. In particular, for any positive root $\alpha \in \Phi^{+}$, we may regard the one-parameter root subgroup $U_{\alpha}$ corresponding to $\alpha$ as a subgroup of $\tilde{G}$.

To any element $w \in W$, the Weyl group, we may define the unipotent subgroup $U_{w}$ by

$$
U_{w}:=\prod_{\alpha \in \Phi^{+}, w(\alpha) \in \Phi^{-}} U_{\alpha} .
$$


Then define the intertwining operator $\mathcal{A}_{w}: I(\chi) \rightarrow I\left({ }^{w} \chi\right)$ by

$$
\mathcal{A}_{w}(f)(g):=\int_{U_{w}} f\left(w^{-1} u g\right) d u
$$

whenever the above integral is absolutely convergent, and by the usual meromorphic continuation in general. (By abuse of notation we are using the same letter $w$ for the Weyl group and for a representative in $K$.)

The representation $\pi_{\mathbf{z}}$ contains a $K$-fixed vector $\phi_{K}$, unique up to constant multiple. We may choose these so that

$$
\mathcal{A}_{w} \phi_{K}^{\mathbf{z}}=c_{w}(\chi) \phi_{K}^{w \mathbf{z}}
$$

where for any simple reflections $s=s_{\alpha}$ and any $w$ such that the length function $\ell\left(s_{\alpha} w\right)=$ $\ell(w)+1$,

$$
c_{s}(\chi)=\frac{1-q^{-1} \mathbf{z}^{n \alpha}}{1-\mathbf{z}^{n \alpha}}, \quad \text { and } \quad c_{s w}(\chi)=c_{s}\left(\chi^{w}\right) c_{w}(\chi) .
$$

Let $\overline{\mathcal{A}}_{w}$ denote the normalized intertwiner:

$$
\overline{\mathcal{A}_{w}}:=c_{w}(\chi)^{-1} \mathcal{A}_{w} .
$$

Let $\psi$ be a character of $U$ such that if $i_{\alpha}$ is the embedding $\mathrm{SL}_{2} \rightarrow \mathrm{GL}_{n}$ along the simple root $\alpha$, then the additive character $x \mapsto i_{\alpha}\left(\begin{array}{c}x \\ 1\end{array}\right)$ of $F$ is trivial on $\mathfrak{o}$ but no larger fractional ideal. A Whittaker functional on a representation $(\pi, V)$ of $\tilde{G}$ is a linear functional $W^{\pi}$ for which

$$
W^{\pi}(\pi(u) v)=\psi(u) W^{\pi}(v) \quad \text { for all } u \in U \text { and } v \in V .
$$

As stated in Section 6 of [35], the dimension of the space of Whittaker functionals for the principal series $I(\chi)$ is equal to the cardinality $n^{r}$ of $\tilde{T} / H$. Let $\mathbf{W}^{\chi}$ denote the $i(\chi)$-valued Whittaker functional on $I(\chi)$ defined by

$$
\mathbf{W}^{\chi}(\phi):=\int_{U^{-}} \phi\left(u w_{0}\right) \overline{\psi(u)} d u: I(\chi) \longrightarrow i(\chi) .
$$

(We denote this $\mathbf{W}^{\mathbf{z}}$ when $\chi=\chi_{\mathbf{z}}$.) Then there is an isomorphism between the linear dual $i(\chi)^{*}$ and the space Whittaker functionals to $\mathbb{C}$ on $I(\chi)$ given by

$$
\mathcal{L} \longmapsto \mathcal{L} \circ W^{\chi}, \quad \text { for } \mathcal{L} \text { in } i(\chi)^{*} .
$$

Let us describe a particular basis of $i(\chi)^{*}$ used in 35 for the computation of the spherical function under the Whittaker functional. Let $v_{0}:=\phi_{K}(1)$, an element of $i(\chi)$. Let $\theta_{\chi}$ denote the representation of $\tilde{T}$ on $i(\chi)$ (denoted $\pi_{\chi}$ in [35]). Then $\left\{\theta_{\chi}\left(\varpi^{\gamma}\right) v_{0} \mid \gamma \in \Gamma\right\}$ is a basis for $i(\chi)$. Let $\left\{\mathcal{L}_{\gamma}^{(\chi)}\right\}$ denote the dual basis of $i(\chi)^{*}$. If $\mu \in \Lambda$ write $\mu=\beta+\gamma$ with $\gamma \in \Gamma$ and $\beta \in n \Lambda$. Then

$$
\mathcal{L}_{\nu}^{(\chi)}\left(\theta_{\chi}\left(\varpi^{\mu}\right) v_{0}\right)= \begin{cases}\chi\left(\varpi^{\beta}\right) & \text { if } \nu=\gamma, \\ 0 & \text { otherwise. }\end{cases}
$$

Thus we obtain a basis of the space of Whittaker functionals on $I(\chi)$, denoted $W_{\gamma}^{\chi}=\mathcal{L}_{\gamma}^{\chi} \circ \mathbf{W}^{\chi}$ using the isomorphism (5.8). We will denote $W_{\gamma}^{\mathbf{z}}=W_{\gamma}^{\chi}$ if $\chi=\chi_{\mathbf{z}}$, or as simply $W_{\gamma}$.

The spherical Whittaker function $W_{\gamma}^{\mathbf{z}}\left(\pi\left(\varpi^{\lambda}\right) \phi_{K}\right)$ vanishes unless the weight $\lambda$ is dominant. One approach to studying them, going back to Casselman and Shalika (for linear groups) and Kazhdan and Patterson for metaplectic covers, is to exploit the fact that $\mathcal{W}^{w} \chi_{\circ} \overline{\mathcal{A}_{w}}$ is an 
$i(\chi)$-valued Whittaker functional for $I(\chi)$. This is the approach that was taken by Chinta and Offen [16] and McNamara [35]. Thus we expand

$$
W_{\mu}^{w \mathbf{z}} \circ \overline{\mathcal{A}}_{w}=\sum_{\nu \in \Gamma} \tau_{\mu, \nu} W_{\nu}^{\mathbf{z}}
$$

for some rational functions $\tau_{\mu, \nu}=\tau_{\mu, \nu}^{(w)}\left(\mathbf{z}^{n}\right)$. It suffices to understand these structure constants on simple reflections $w=s_{\alpha}$. These were computed for metaplectic covers of $\operatorname{GL}(r)$ by Kazhdan and Patterson, and we discuss their calculation following Theorem 13.1 in [35].

We now introduce the Gauss sums $g(a)$, which depend on $a$ modulo $n$ and satisfy the conditions $g(0)=-v$, while $g(a) g(n-a)=v$ if $n$ does not divide $a$, with $v=q^{-1}$. These are given by the formula

$$
g(a)=\frac{1}{q} \sum_{t \in(\mathfrak{o} /(\varpi))^{\times}}(\varpi, t)^{a} \psi\left(\frac{t}{\varpi}\right) .
$$

Proposition 5.1 (Kazhdan-Patterson, [26], Lemma I.3.3). Let $s=s_{\alpha}$ be a simple reflection and let $\mu, \nu \in \Gamma$. The structure constants $\tau_{\nu, \mu}:=\tau_{\nu, \mu}^{(w)}$ for can be broken into two pieces:

$$
\tau_{\nu, \mu}=\tau_{\nu, \mu}^{1}+\tau_{\nu, \mu}^{2}
$$

where $\tau^{1}$ vanishes unless $\nu \sim \mu \bmod n \Lambda$ and $\tau^{2}$ vanishes unless $\nu \sim s(\mu)+\alpha \bmod n \Lambda$. Moreover:

$$
\tau_{\mu, \mu}^{1}=\left(1-q^{-1}\right) \frac{\mathbf{z}^{n\left\lceil\frac{\langle\alpha, \mu\rangle}{n}\right\rceil \alpha}}{1-q^{-1} \mathbf{z}^{n \alpha}}
$$

where $\lceil x\rceil$ denotes the smallest integer at least $x$, and

$$
\tau_{s(\mu)+\alpha, \mu}^{2}=g(\langle\alpha, \mu-\rho\rangle) \frac{1-\mathbf{z}^{n \alpha}}{1-q^{-1} \mathbf{z}^{n \alpha}} .
$$

Proof. This is Theorem 13.1 in [35]. Recall that $Q(\alpha)=1$ on simple roots $\alpha$ so $n_{\alpha}=$ $n / \operatorname{gcd}(n, Q(\alpha))=n$. Our cocycle has been chosen so that $B(\alpha, \mu)=\langle\alpha, \mu\rangle$. Our $n$-th order Gauss sum $g$ is $q^{-1} \mathfrak{g}$ in the notation of [35]. Finally, we have $x_{\alpha}=\mathbf{z}^{\alpha}$ to obtain (5.12) and (5.13).

We now return to the $R$-matrices. We will not be concerned with partition functions in this section but we will use the notation of Boltzmann weights (slightly modified) in order to make a connection with Proposition 5.1.

The weights we need now are given in Figures 5 and 6; we will derive these from those in Figures 2 and 3. We remind the reader that in Section 2, we stressed that it suffices to consider only the decoration 0 associated to a - spin, and the table below reflects this assumption. So we omit other $-a$ decorated spins with $a \neq 0$.

Proposition 5.2. The Yang-Baxter equation is satisfied with the weights in Figures 5 and 6.

Proof. To obtain these from the weights in Figure 2 and 3 , we make use of the change of basis method described in Section 4 . We take the function $f(\alpha, z)$ to equal

$$
\begin{cases}z^{a} & \text { if } \alpha=+a, 0 \leqslant a<n, \\ 1 & \text { if } \alpha=-0 .\end{cases}
$$


In Figure 5 we further divide each weight by $z_{i}$, and in Figure 6 we divide by $z_{1}^{n}-v z_{2}^{n}$. These multiplications apply to all weights, so we may do this at our convenience without affecting the Yang-Baxter equation.

\begin{tabular}{|c|c|c|c|c|c|}
\hline$a_{1}$ & $a_{2}$ & $\mathrm{~b}_{1}$ & $\mathrm{~b}_{2}$ & $\mathrm{C}_{1}$ & $\mathrm{C}_{2}$ \\
\hline${ }_{z_{i}^{-n \delta(a+1)}}^{a+1 \overbrace{\oplus}^{a}}$ & $\stackrel{0}{\ominus} \bigodot_{1}^{\Theta} \overbrace{-}^{0}$ & $\begin{array}{c}a+1 \odot a \\
\oplus(a) z_{i}^{-n \delta(a+1)}\end{array}$ & $\stackrel{0}{\ominus} \underset{\oplus}{\oplus} \stackrel{0}{\Theta}$ & $\stackrel{0}{\ominus} \underset{-}{\oplus} \stackrel{0}{\oplus}$ & $\stackrel{1}{\oplus} \overbrace{z_{i}^{-n \delta(1)}}^{-} 0$ \\
\hline
\end{tabular}

Figure 5. Modified Boltzmann weights.

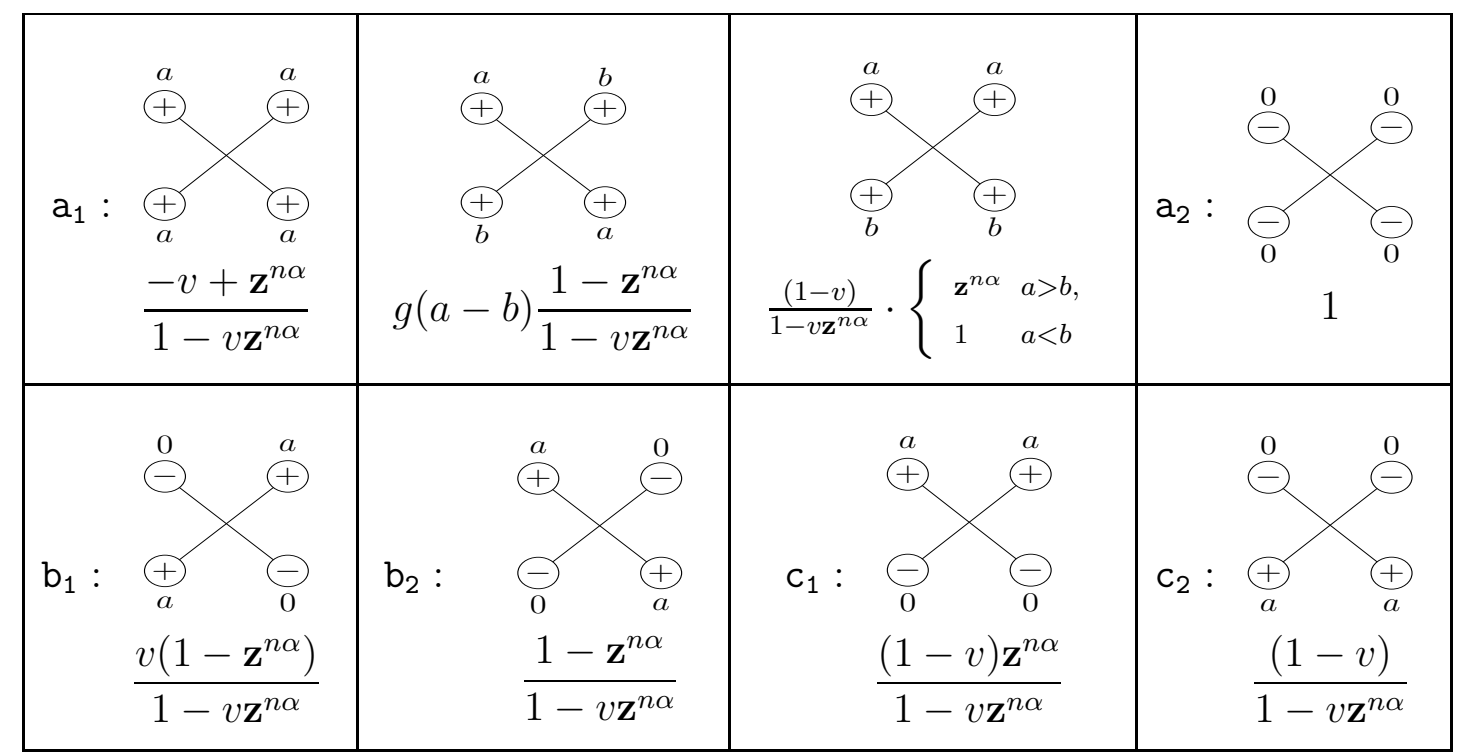

Figure 6. Modified weights $\hat{R}_{\mathbf{z}}$. When combined with the weights in Figure5, they satisfy a Yang-Baxter equation. This follows from Theorem 3.1. In this figure we are assuming that charges depicted as $a$ and $b$ are in distinct residue classes $\bmod n$.

Proposition 5.3. Let $\mu \in X_{*}(T) \simeq \mathbb{C}[\Lambda]$ with $\mu-\rho=\left(c_{1}, \cdots, c_{r}\right)$ for some integers $c_{i} \in[0, n)$. Let $\tau_{\nu, \mu}(\mathbf{z}):=\tau_{\nu, \mu}^{\left(s_{i}\right)}(\mathbf{z})$ as in Proposition 5.1. Let wt be the weights for $\hat{R}$ in Figure [6] above with $v=q^{-1}$. Given any pair of integers $a, b$ with $a \equiv c_{i}$ and $b \equiv c_{i+1} \bmod$ $n$, if $a \not \equiv b \bmod n$, then

$$
\tau_{\mu, \mu}^{1}(\mathbf{z})=\mathrm{wt}(\overbrace{a}^{\oplus} \overbrace{a}^{b}) \text { and } \tau_{s_{i}(\mu)+\alpha_{i}, \mu}^{2}(\mathbf{z})=\mathrm{wt}(\overbrace{b}^{\oplus} \overbrace{a}^{+}) \text {. }
$$


If $a \equiv b \bmod n$, then both $\tau_{\mu, \mu}^{1}(\mathbf{z})$ and $\tau_{s_{i}(\mu)+\alpha_{i}, \mu}^{2}(\mathbf{z})$ are nonzero and

$$
\left.\tau_{\mu, \mu}^{1}(\mathbf{z})+\tau_{s_{i}(\mu)+\alpha_{i}, \mu}^{2}(\mathbf{z})=\mathrm{wt}(\oplus_{a}^{\oplus} \overbrace{a}^{+}) \stackrel{a}{\oplus}\right) \text {. }
$$

Proof. We begin by rewriting $\tau^{1}$ and $\tau^{2}$ in terms of $c_{i}$ and $c_{i+1}$. Recall from (5.12) that with $\alpha=\alpha_{i}$

$$
\begin{aligned}
\tau_{\mu, \mu}^{1}=\left(\left(1-q^{-1}\right) \frac{\mathbf{z}^{n\left\lceil\frac{\langle\alpha, \mu\rangle}{n}\right\rceil \alpha}}{1-q^{-1} \mathbf{z}^{n \alpha}}\right. & =\left(1-q^{-1}\right) \frac{\mathbf{z}^{n\left\lceil\frac{c_{i}-c_{i+1}+1}{n}\right\rceil \alpha}}{1-q^{-1} \mathbf{z}^{n \alpha}} \\
& =\frac{\left(1-q^{-1}\right)}{1-q^{-1} \mathbf{z}^{n \alpha}} \begin{cases}\mathbf{z}^{n \alpha} & \text { if } c_{i}-c_{i+1} \geqslant 0 \\
1 & \text { if } c_{i}-c_{i+1}<0 .\end{cases}
\end{aligned}
$$

where the second equality used that $\langle\alpha, \mu-\rho\rangle=c_{i}-c_{i+1}$. From (5.13),

$$
\tau_{s(\mu)+\alpha, \mu}^{2}=g(\langle\alpha, \mu-\rho\rangle) \frac{1-\mathbf{z}^{n \alpha}}{1-q^{-1} \mathbf{z}^{n \alpha}}=g\left(c_{i}-c_{i+1}\right) \frac{1-\mathbf{z}^{n \alpha}}{1-q^{-1} \mathbf{z}^{n \alpha}} .
$$

Note that $\mu=s_{i}(\mu)+\alpha_{i}$ if and only if $c_{i}=c_{i+1}$, since $s_{i}(\mu)+\alpha_{i}=s_{i}(\mu-\rho)+\rho$, so $\tau^{1}$ and $\tau^{2}$ are only simultaneously nonzero when $c_{i}=c_{i+1}$.

Now we compare to the weights in Figure 6 with $a-b \equiv c_{i+1}-c_{i}(\bmod n)$ according to cases. First if $c_{i} \neq c_{i+1}$ so that $a \not \equiv b(\bmod n)$, then the modified $R$-vertex weight in the top row, third column entry of Figure 6 indeed matches the evaluation of $\tau^{1}$ above upon setting $v=q^{-1}$. Moreover, the top row, second column entry of Figure 6 agrees with $\tau^{2}$ under the same specialization $v=q^{-1}$.

To finish, consider the case when $c_{i}=c_{i+1}$ so that both $\tau^{1}$ and $\tau^{2}$ are nonzero. Then

$$
\tau_{\mu, \mu}^{1}+\tau_{s(\mu)+\alpha, \mu}^{2}=\frac{\left(1-q^{-1}\right) \mathbf{z}^{n \alpha}}{1-q^{-1} \mathbf{z}^{n \alpha}}-q^{-1} \frac{1-\mathbf{z}^{n \alpha}}{1-q^{-1} \mathbf{z}^{n \alpha}}=\frac{\mathbf{z}^{n \alpha}-q^{-1}}{1-q^{-1} \mathbf{z}^{n \alpha}}
$$

since $g(0)=-v=-q^{-1}$. And this in turn is precisely the weight of the $R$-vertex in the top row, first column of Figure 6 where $a \equiv c_{i}=c_{i+1}$ and $v=q^{-1}$.

Recall that the module of Whittaker coinvariants $\pi_{\mathbf{z}, \psi}$ is naturally the dual space of the space $\mathcal{W}^{\mathbf{z}}$ of Whittaker functionals on $\pi_{\mathbf{z}}$. If $\gamma \in \Gamma$, let $\Omega_{\mu}$ be the image of $\mathbf{s}\left(\varpi^{\mu}\right)$ in $\pi_{\mathbf{z}, \psi}$. Using (5.9) this $\left\{\Omega_{\mu}\right\}$ is the basis of $\pi_{\mathbf{z}, \psi}$ dual to the basis $W_{\mu}^{\mathbf{z}}$ of $\mathcal{W}^{\mathbf{z}}$. Then the map $\overline{\mathcal{A}}_{s_{i}}$ induces the map

$$
\overline{\mathcal{A}}_{s_{i}}\left(\Omega_{\mu}\right)=\sum_{\nu} \tau_{\nu, \mu} \Omega_{\nu}
$$

This follows from (5.10) by duality. We define the map $\theta_{\mathbf{z}}: \pi_{\mathbf{z}, \psi} \longrightarrow \bigoplus_{i} V_{+}\left(z_{i}\right)$ (needed for Theorem 1.1) by

$$
\theta_{\mathbf{z}}\left(\Omega_{\mu}\right)=v_{c_{1}} \otimes \cdots \otimes v_{c_{r}}
$$

when $\mu \in \Gamma$. Recall that this means $\mu-\rho=\left(c_{1}, \cdots, c_{r}\right)$ with $0 \leqslant c_{i}<n$. We will use the notation $v_{\mu-\rho}$ to denote this vector.

We are now ready to prove one of our main results. 
Proof of Theorem 1.1. Let $\mu \in \Gamma$. Let $\nu=s_{i} \mu+\alpha$. Write $\mu-\rho=\left(c_{1}, \cdots, c_{r}\right)$ and $\nu=s_{i} \mu+\alpha$, so that $\nu-\rho=s_{i}(\mu-\rho)$ has the same components with $c_{i}$ and $c_{i+1}$ interchanged.

We consider the case where $\mu \neq \nu$. We have

$$
\theta_{s_{i} \mathbf{z}} \overline{\mathcal{A}}_{s_{i}}\left(\Omega_{\mu}\right)=\theta\left(\tau_{\mu, \mu} \Omega_{\mu}+\tau_{\nu, \mu} \Omega_{\nu}\right)=\tau_{\mu, \mu} v_{\mu-\rho}+\tau_{\nu, \mu} v_{\nu-\rho} .
$$

On the other hand using (4.1)

$$
\tau R\left(v_{c_{i}} \otimes v_{c_{j}}\right)=\sum_{c_{k}, c_{l}} R_{c_{i}, c_{j}}^{c_{k}, c_{l}}\left(v_{c_{l}} \otimes v_{c_{k}}\right)
$$

Taking $j=i+1$, on the right-hand side, the only nonzero terms are $\left(c_{l}, c_{k}\right)=\left(c_{i}, c_{i+1}\right)$ or $\left(c_{i+1}, c_{i}\right)$. So

$$
(\tau R)_{i, i+1} \theta_{\mathbf{z}}\left(\Omega_{\mu}\right)=\left(R_{c_{i}, c_{i+1}}^{c_{i+1}, c_{i}}\right)_{i, i+1} v_{\mu-\rho}+\left(R_{c_{i}, c_{i+1}}^{c_{i}, c_{i+1}}\right)_{i, i+1} v_{\nu-\rho}
$$

(The subscript $X_{i, i+1}$ means that the operator is applied in the $i, i+1$ position of the $r$-fold tensor product $V_{+\mathbf{z}} \cdot$ ) Thus we need

$$
\left(R_{c_{i}, c_{i+1}}^{c_{i+1}, c_{i}}\right)_{i, i+1}=\tau_{\mu, \mu}, \quad\left(R_{c_{i}, c_{i+1}}^{c_{i}, c_{i+1}}\right)_{i, i+1}=\tau_{\nu, \mu}
$$

and this is the content of Proposition 5.3 .

The case where $\mu=\nu$ is similar, using (5.14).

\section{Functional Equations via Partition Functions}

In Theorem 2.5, we gave one spherical Whittaker function as the partition function of a solvable lattice model. However there are $n^{r}$ independent Whittaker functions. In this section, we will show that the charge statistic can be refined to give $n^{r}$ independent Whittaker functions. In this section we will use the unmodified weights in Figure 2 ,

In Theorem 2.5, we were vague as to the precise normalization. Now that we have defined enough notation in the previous section, let us give the precise normalization. Let $\chi=\chi_{\mathbf{z}}$. There is a unique functional $\mathcal{L}^{\circ}$ on $i(\chi)$ such that

$$
\mathcal{L}^{\circ}\left(\theta\left(\varpi^{\lambda}\right) v_{0}\right)=\mathbf{z}^{\lambda} .
$$

(This was denoted $\lambda$ in [33], which underlies the proof of Theorem 1.1.) Evidently

$$
\mathcal{L}^{\circ}=\sum_{\gamma \in \Gamma} \mathbf{z}^{\gamma} \mathcal{L}_{\gamma}
$$

where $\Gamma$ is the set of representatives defined in (5.4). We define, for $g \in \tilde{G}$

$$
W^{\circ}(g)=\mathcal{L}^{\circ} \mathbf{W}\left(\pi(g) \phi_{K}\right),
$$

where $\mathbf{W}$ is defined in (5.7). The correct normalization for Theorem 2.5 is:

$$
Z\left(\mathfrak{S}_{\lambda}\right)=\mathbf{z}^{w_{0} \rho} \delta^{-1 / 2}\left(\varpi^{\lambda}\right) W^{\circ}\left(\varpi^{\lambda}\right) .
$$

Now if $\gamma \in \Gamma$ then

$$
W_{\gamma}(g)=\mathcal{L}_{\gamma} W\left(\pi(g) \phi_{K}\right)
$$

so that $W^{\circ}=\sum \mathbf{z}^{\gamma} W_{\gamma}$.

Let $\mathfrak{M}=\mathbb{C}\left(z_{1}, \cdots, z_{r}, v\right)$ be the field of rational functions in $z_{i}$ and $v$, which we may think of as indeterminates. Let $\mathfrak{M}_{n}=\mathbb{C}\left(z_{1}^{n}, \cdots, z_{r}^{n}, v\right)$. Then $\left[\mathfrak{M}: \mathfrak{M}_{n}\right]=n^{r}$ and a basis of $\mathfrak{M}$ over $\mathfrak{M}_{n}$ consists of the $n^{r}$ elements $\mathbf{z}^{\gamma}$ where $\gamma \in \Gamma$, the set of representatives defined by (5.4). 
Lemma 6.1. Let $g \in \tilde{G}$ and let $\gamma \in \Gamma$. Then $\mathcal{L}_{\gamma}\left(\pi(g) \phi_{K}\right)$ is in $\mathfrak{M}_{n}$.

Proof. We make an Iwasawa decomposition $g=\varepsilon t v \varpi^{\nu} k$ where $\varepsilon \in \mu_{n}, t \in T(\mathfrak{o}), u \in U$, $\nu \in \Lambda$ and $k \in K$. Then $\mathcal{L}_{\gamma}\left(\pi(g) \phi_{K}\right)=\varepsilon \mathcal{L}\left(\pi\left(\varpi^{\nu}\right) \phi_{K}\right)=\varepsilon \mathcal{L}_{\gamma}\left(\theta\left(\varpi^{\lambda}\right) v_{0}\right)$. This depends only $\mathbf{z}^{n}$ by (15.9).

Proposition 6.2. Let $\lambda$ be a dominant weight, and let $\gamma \in \Gamma$. Then the function $\mathbf{z}^{\gamma} W_{\gamma}^{\mathbf{z}}\left(\varpi^{\lambda} \phi_{K}\right)$, considered as a function of $\mathbf{z}$, lies in the coset $\mathbf{z}^{\gamma} \mathfrak{M}_{n}$.

Proof. The function $W_{\gamma}^{\mathbf{z}}\left(\varpi^{\lambda} \phi_{K}\right)$ is defined by the integral

$$
\int_{U_{-}} \mathcal{L}_{\gamma}^{\mathbf{z}} \phi_{K}\left(u w_{0} \varpi^{\lambda}\right) \overline{\psi(u)} d u
$$

For every value of $u$ the integrand is in $\mathfrak{M}_{n}$ by Lemma 6.1. Hence the integral is, also.

If $x \in \mathbb{Z}$ let $[x]$ denote the least residue of $x$ modulo $n$. The following result is a refinement of Theorem 2.5.

Theorem 6.3. Let $\left(c_{1}, \cdots, c_{r}\right) \in \mathbb{Z}^{r}$ and let $\gamma \in \Gamma$ be defined by $\gamma_{i}-r+i=\left[N+1-r-c_{i}\right]$. Then

$$
Z\left(\mathfrak{S}_{\lambda} ; \mathbf{c}\right)=\mathbf{z}^{w_{0} \rho+\gamma} \delta^{-1 / 2}\left(\varpi^{\lambda}\right) W_{\gamma}\left(\varpi^{\lambda}\right)
$$

Proof. Since as a vector space $\mathfrak{M}=\bigoplus_{\gamma \in \Gamma} \mathbf{z}^{\gamma} \mathfrak{M}_{n}$, we may project both sides in (6.2) onto $\mathbf{z}^{\gamma+w_{0} \rho} \mathfrak{M}_{n}$. It follows from Proposition 6.2 that the projection of the right-hand side is $\mathbf{z}^{\gamma+w_{0} \rho} \delta^{-1 / 2}\left(\varpi^{\lambda}\right) W_{\gamma}\left(\varpi^{\lambda}\right)$. As for the left-hand side consider a state $\mathfrak{s}$ of the system $\mathfrak{S}_{\lambda}$. We observe that in the Boltzmann weights in Figure 2 the vertex contributes a $z_{i}$ to the partition function if the spin to the left of the vertex is - , while it increments the charge if the spin to the left is + . Thus let $c_{i}$ be the charge at the left edge in the $i$-th row, and let $\nu_{i}$ be the power of $z_{i}$ that appears in the Boltzmann weight of the state. We see that $c_{i}+\nu_{i}=N$. Now if the Boltzmann weight of the state is in $\mathbf{z}^{\gamma+w_{0} \rho} \mathfrak{M}_{n}$ we must have $\nu_{i} \equiv \gamma_{i}+i-1$ modulo $N$. Now $\gamma_{i}-\rho_{i}=\gamma_{i}-r+i \equiv \nu_{i}-r+1 \equiv N+1-r-c_{i}$. Remembering that $\gamma \in \Gamma$ means that $0 \leqslant \gamma_{i}-\rho_{i}<n$, we see that the state $\mathfrak{s}$ contributes to $Z\left(\mathfrak{S}_{\lambda} ; \mathbf{c}\right)$ if and only if $\gamma_{i}-r+i=\left[N+1-r-c_{i}\right]$ and the statement follows.

Our next result is a variant of Theorem 1.1 that describes the functional equations of the partition function. In view of (6.2), this can also be regarded as a functional equation for the Whittaker functions. We will add $\mathbf{z}$ to the notation and denote $Z\left(\mathfrak{S}_{\lambda} ; \mathbf{c}\right)=Z\left(\mathfrak{S}_{\lambda, \mathbf{z}} ; \mathbf{c}\right)$. Let $s_{i}$ be the simple reflection that interchanges $i$ and $i+1$. In our notation, note that $\mathbf{z}^{\alpha_{i}}=z_{i} / z_{i+1}$, where $\alpha_{i}$ is the $i$-th simple root.

Proposition 6.4. Let $c$ be the least residue of $c_{i}-c_{i+1}$ modulo $n$. Then

$$
Z\left(\mathfrak{S}_{\lambda, s_{i} \mathbf{z}} ; s_{i} \mathbf{c}\right)=(1-v) \frac{\mathbf{z}^{(n-c) \alpha_{i}}}{1-v \mathbf{z}^{n \alpha}} Z\left(\mathfrak{S}_{\lambda, \mathbf{z}} ; s_{i} \mathbf{c}\right)+g\left(c_{i}-c_{i+1}\right) \frac{1-\mathbf{z}^{n \alpha}}{1-v \mathbf{z}^{n \alpha}} Z\left(\mathfrak{S}_{\lambda, \mathbf{z}} ; \mathbf{c}\right)
$$


Proof. We attach the vertex $R_{z_{i}, z_{i+1}}$ to the right of the partition function of the system $\left(\mathfrak{S}_{\lambda, s_{i} \mathbf{z}} ; s_{i} \mathbf{c}\right)$, thus:

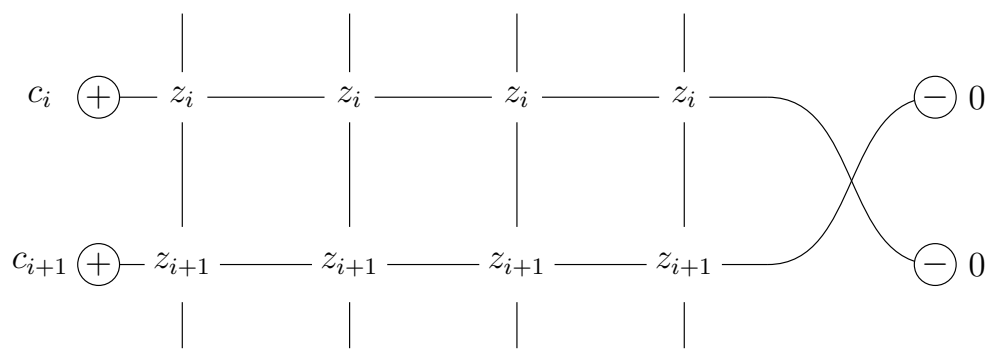

Consulting Figure 3, there is only one possible configuration for the $R$-vertex, so attaching it just multiplies the partition function by $z_{i+1}^{n}-v z_{i}^{n}$. Now using the Yang-Baxter equation we may move the $R$-matrix to the left, and obtain the partition function of a system that looks like this:

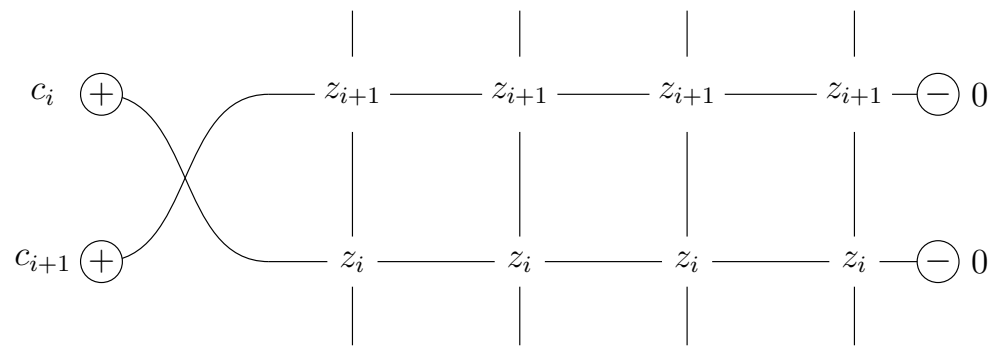

In this case there are two possibilities for the charges on the edges to the right of the $R$-vertex (unless $c_{i} \equiv c_{i+1}$ modulo $n$ ) and the two terms may be found again in Figure 3 .

It must be checked that the statement remains true if $c_{i}=c_{i+1}$ though in this case the two terms on the right-hand side in (6.3) may be combined. Since $g(0)=-v$ the coefficient is

$$
\frac{(1-v) \mathbf{z}^{n \alpha_{i}}}{1-v \mathbf{z}^{n \alpha_{i}}}+\frac{-v\left(1-\mathbf{z}^{n \alpha_{i}}\right)}{1-v \mathbf{z}^{n \alpha_{i}}}=\frac{-v+\mathbf{z}^{n \alpha_{i}}}{1-v \mathbf{z}^{n \alpha_{i}}} .
$$

This is what we want by Figure 3 .

Consider the following version of equation (6.3):

$$
\widetilde{Z}\left(\mathfrak{S}_{\lambda, s_{i} \mathbf{z}} ; s_{i} \mathbf{c}\right)=\widetilde{\mathrm{wt}}(\overbrace{c_{i}}^{c_{\oplus}^{c_{i+1}}} \overbrace{c_{i+1}}^{c_{i}}) \widetilde{Z}\left(\mathfrak{S}_{\lambda, \mathbf{z}} ; s_{i} \mathbf{c}\right)+\widetilde{\mathrm{wt}}(\overbrace{c_{i}}^{\oplus} \overbrace{c_{i}}^{c_{i+1}}) \widetilde{Z}\left(\mathfrak{S}_{\lambda, \mathbf{z}} ; \mathbf{c}\right) \text {, }
$$

where now $\widetilde{Z}$ is the partition function of the same ice model as before, but using the modified weights from Figure 5, and $\widetilde{\mathrm{wt}}$ are also the modified weights from Figure 6 .

This may be compared with a result from Section 5. Let $\varpi^{\nu}$ be a representative in $\tilde{T} / H$ with $\nu-\rho=\left(c_{1}, \cdots, c_{r}\right)$ with $c_{i} \in[0, n)$. Then for a simple reflection $s_{i}$ the following functional equation holds as explained in Proposition 5.1;

$$
W_{\nu}^{s_{i} \chi} \circ \overline{\mathcal{A}}_{s_{i}}\left(\pi\left(\varpi^{\lambda}\right) \phi_{K}\right)=\tau_{\nu, \nu}^{1} W_{\nu}^{\chi}\left(\pi\left(\varpi^{\lambda}\right) \phi_{K}\right)+\tau_{\nu, s_{i} \cdot \nu}^{2} W_{s_{i} \nu}^{\chi}\left(\pi\left(\varpi^{\lambda}\right) \phi_{K}\right) .
$$

Remark 6.5. Combining the results of Proposition 5.3 and Theorem 6.3, we immediately conclude that the right hand sides of (6.4) and of (6.5) are equal. We therefore obtain an interpretation of the action of the intertwining operator on the Whittaker function at the 
ice model level. To be more precise, the effect of the intertwining operator $\overline{\mathcal{A}}_{s_{1}}$ on spherical Whittaker functions is realized by swapping the roles of the parameters $z_{i}$ and $z_{i+1}$ in the ice model while attaching an $R$-matrix at the edge of the system. The effect of this operation is described by the Boltzmann weights of the attached $R$-matrix.

This shows that the functional equation of the Whittaker function has an interpretation as equality of partition functions. Such equivalences are useful when they transform a hard problem in one area to an easy problem in a different area. Another example of such an equivalence is given in [6]. There, we (together with Gray) prove the equality of the partition functions of two ice models which is equivalent to the equality of two expressions for coefficients of Weyl group multiple Dirichlet series. The proof of this fact occupies most of [11; the proof is long and intricate. The proof in [6] on the other hand is very short and clear.

\section{REFERENCES}

[1] William D. Banks, Jason Levy, and Mark R. Sepanski. Block-compatible metaplectic cocycles. J. Reine Angew. Math., 507:131-163, 1999.

[2] Rodney J. Baxter. Exactly solved models in statistical mechanics. Academic Press Inc. [Harcourt Brace Jovanovich Publishers], London, 1982.

[3] V. V. Bazhanov and A. G. Shadrikov. Trigonometric solutions of the triangle equations, and simple Lie superalgebras. Teoret. Mat. Fiz., 73(3):402-419, 1987.

[4] Ben Brubaker, Valentin Buciumas, and Daniel Bump. arXiv: 1604.02206v2 (earlier draft of this paper), 2016.

[5] Ben Brubaker, Valentin Buciumas, Daniel Bump, and Solomon Friedberg. Hecke modules from metaplectic ice. Selecta Math. (N.S.), 2017, arXiv:1704.00701.

[6] Ben Brubaker, Valentin Buciumas, Daniel Bump, and Nathan Gray. Duality for metaplectic ice, 2017, arXiv:1709.06500.

[7] Ben Brubaker, Valentin Buciumas, Daniel Bump, and Henrik Gustafsson. Vertex operators, solvable lattice models and metaplectic Whittaker functions, 2018, arXiv:1806.07776.

[8] Ben Brubaker, Daniel Bump, Gautam Chinta, Solomon Friedberg, and Paul E. Gunnells. Metaplectic ice. In Multiple Dirichlet series, L-functions and automorphic forms, volume 300 of Progr. Math., pages 65-92. Birkhäuser/Springer, New York, 2012.

[9] Ben Brubaker, Daniel Bump, and Solomon Friedberg. Schur polynomials and the Yang-Baxter equation. Comm. Math. Phys., 308(2):281-301, 2011.

[10] Ben Brubaker, Daniel Bump, and Solomon Friedberg. Weyl group multiple Dirichlet series, Eisenstein series and crystal bases. Ann. of Math. (2), 173(2):1081-1120, 2011.

[11] Ben Brubaker, Daniel Bump, and Solomon Friedberg. Weyl group multiple Dirichlet series: type A combinatorial theory, volume 175 of Annals of Mathematics Studies. Princeton University Press, Princeton, NJ, 2011.

[12] Ben Brubaker and Andrew Schultz. The six-vertex model and deformations of the Weyl character formula. J. Algebraic Combin., 42(4):917-958, 2015.

[13] Jean-Luc Brylinski and Pierre Deligne. Central extensions of reductive groups by $\mathbf{K}_{2}$. Publ. Math. Inst. Hautes Études Sci., (94):5-85, 2001.

[14] W. Casselman and J. Shalika. The unramified principal series of $p$-adic groups. II. The Whittaker function. Compositio Math., 41(2):207-231, 1980.

[15] Vyjayanthi Chari and Andrew Pressley. A guide to quantum groups. Cambridge University Press, Cambridge, 1994.

[16] Gautam Chinta and Omer Offen. A metaplectic Casselman-Shalika formula for $\mathrm{GL}_{r}$. Amer. J. Math., 135(2):403-441, 2013.

[17] V. G. Drinfeld. Quantum groups. In Proceedings of the International Congress of Mathematicians, Vol. 1, 2 (Berkeley, Calif., 1986), pages 798-820. Amer. Math. Soc., Providence, RI, 1987. 
[18] V. G. Drinfeld. Quasi-Hopf algebras. Algebra i Analiz, 1(6):114-148, 1989.

[19] I. B. Frenkel and N. Yu. Reshetikhin. Quantum affine algebras and holonomic difference equations. Comm. Math. Phys., 146(1):1-60, 1992.

[20] D. Gaitsgory. Twisted Whittaker model and factorizable sheaves. Selecta Math. (N.S.), 13(4):617-659, 2008.

[21] D. Gaitsgory and S. Lysenko. Parameters and duality for the metaplectic geometric Langlands theory. Selecta Math. (N.S.), 24(1):227-301, 2018, arXiv:1608.00284.

[22] Nathan Gray. Metaplectic ice for Cartan type C, 2017, arXiv:1709.04971.

[23] Michio Jimbo. A $q$-difference analogue of $U(\mathfrak{g})$ and the Yang-Baxter equation. Lett. Math. Phys., 10(1):63-69, 1985.

[24] Michio Jimbo. Quantum $R$ matrix related to the generalized Toda system: an algebraic approach. In Field theory, quantum gravity and strings (Meudon/Paris, 1984/1985), volume 246 of Lecture Notes in Phys., pages 335-361. Springer, Berlin, 1986.

[25] M. Kashiwara, T. Miwa, and E. Stern. Decomposition of $q$-deformed Fock spaces. Selecta Math. (N.S.), 1(4):787-805, 1995.

[26] D. A. Kazhdan and S. J. Patterson. Metaplectic forms. Inst. Hautes Études Sci. Publ. Math., (59):35$142,1984$.

[27] Takeo Kojima. Diagonalization of transfer matrix of supersymmetry $U_{q}(\widehat{s l}(M+1 \mid N+1))$ chain with a boundary. J. Math. Phys., 54(4), 2013.

[28] Tomio Kubota. On automorphic functions and the reciprocity law in a number field. Lectures in Mathematics, Department of Mathematics, Kyoto University, No. 2. Kinokuniya Book-Store Co., Ltd., Tokyo, 1969.

[29] P. P. Kulish, N. Yu. Reshetikhin, and E. K. Sklyanin. Yang-Baxter equations and representation theory. I. Lett. Math. Phys., 5(5):393-403, 1981.

[30] Elliott Lieb. Exact solution of the problem of entropy in two-dimensional ice. Phys. Rev. Lett., 18:692694, 1967.

[31] Sergey Lysenko. Twisted Whittaker models for metaplectic groups. Geom. Funct. Anal., 27(2):289-372, 2017, arXiv:1509.02433.

[32] Hideya Matsumoto. Sur les sous-groupes arithmétiques des groupes semi-simples déployés. Ann. Sci. École Norm. Sup. (4), 2:1-62, 1969.

[33] Peter J. McNamara. Metaplectic Whittaker functions and crystal bases. Duke Math. J., 156(1):1-31, 2011.

[34] Peter J. McNamara. Principal series representations of metaplectic groups over local fields. In Multiple Dirichlet series, L-functions and automorphic forms, volume 300 of Progr. Math., pages 299-327. Birkhäuser/Springer, New York, 2012.

[35] Peter J. McNamara. The metaplectic Casselman-Shalika formula. Trans. Amer. Math. Soc., 368(4):2913-2937, 2016.

[36] Jürgen Neukirch. Algebraic number theory, volume 322 of Grundlehren der Mathematischen Wissenschaften [Fundamental Principles of Mathematical Sciences]. Springer-Verlag, Berlin, 1999. Translated from the 1992 German original and with a note by Norbert Schappacher, With a foreword by G. Harder.

[37] Jacques H. H. Perk and Cherie L. Schultz. New families of commuting transfer matrices in $q$-state vertex models. Phys. Lett. A, 84(8):407-410, 1981.

[38] Anna Puskás. Whittaker functions on metaplectic covers of $G L(r), 2016$, arXiv:1605.05400.

[39] N. Reshetikhin. Multiparameter quantum groups and twisted quasitriangular Hopf algebras. Lett. Math. Phys., 20(4):331-335, 1990.

[40] Bill Sutherland. Exact solution for a model for hydrogen-bonded crystals. Phys. Rev. Lett., 19(3):103-4, 1967.

[41] Martin H. Weissman. Split metaplectic groups and their L-groups. J. Reine Angew. Math., 696:89-141, 2014.

[42] Hiroyuki Yamane. Quantized enveloping algebras associated with simple Lie superalgebras and their universal R-matrices. Publ. Res. Inst. Math. Sci., 30(1):15-87, 1994. 
[43] Huafeng Zhang. Fundamental representations of quantum affine superalgebras and $R$-matrices. Transform. Groups, 22(2):559-590, 2017, arxiv:1506.06093.

[44] R. B. Zhang. Structure and representations of the quantum general linear supergroup. Comm. Math. Phys., 195(3):525-547, 1998.

School of Mathematics, University of Minnesota, Minneapolis, MN 55455

E-mail address: brubaker@math.umn.edu

Einstein Institute of Mathematics, Edmond J. Safra Campus, Givat Ram, The Hebrew University of Jerusalem, Jerusalem, 91904, IsRael

E-mail address: valentin.buciumas@gmail.com

Department of Mathematics, Stanford University, Stanford, CA 94305-2125

E-mail address: bump@math.stanford.edu 